\title{
Targeted delivery of polyamidoamine-paclitaxel conjugate functionalized with anti-human epidermal growth factor receptor 2 trastuzumab
}

This article was published in the following Dove Press journal:

International Journal of Nanomedicine

18 March 2015

Number of times this article has been viewed

\section{Pengkai Ma' \\ Xuemei Zhang' \\ Ling $\mathrm{Ni}^{2}$ \\ Jinming $\mathrm{Li}^{2}$ \\ Fengpu Zhang' \\ Zheng Wang' \\ Shengnan Lian' \\ Kaoxiang Sun'}

'School of Pharmacy, Yantai University, Yantai, Shandong Province, People's

Republic of China; ${ }^{2}$ State Key

Laboratory of Long-acting and

Targeting Drug Delivery System,

Yantai, Shandong Province, People's

Republic of China
Correspondence: Kaoxiang Sun School of Pharmacy, Yantai University, No 32 Qingquan Road, Yantai, 264005 Shandong Province, People's Republic of China

Tel +865353808266

Emailsunkx@ytu.edu.cn
Background: Antibody-dendrimer conjugates have the potential to improve the targeting and release of chemotherapeutic drugs at the tumor site while reducing adverse side effects caused by drug accumulation in healthy tissues. In this study, trastuzumab (TMAB), which binds to human epidermal growth factor receptor 2 (HER2), was used as a targeting agent in a TMAB-polyamidoamine (PAMAM) conjugate carrying paclitaxel (PTX) specifically to cells overexpressing HER2.

Methods: TMAB was covalently linked to a PAMAM dendrimer via bifunctional polyethylene glycol (PEG). PTX was conjugated to PAMAM using succinic anhydride as a cross-linker, yielding TMAB-PEG-PAMAM-PTX. Dynamic light scattering and transmission electron microscopy were used to characterize the conjugates. The cellular uptake and in vivo biodistribution were studied by fluorescence microscopy, flow cytometry, and Carestream In Vivo FX, respectively.

Results: Nuclear magnetic resonance spectroscopy demonstrated that PEG, PTX, fluorescein isothiocyanate, and cyanine7 were conjugated to PAMAM. Ultraviolet-visible spectroscopy and sodium dodecyl sulfate polyacrylamide gel electrophoresis demonstrated that TMAB was conjugated to PEG-PAMAM. Dynamic light scattering and transmission electron microscopy measurements revealed that the different conjugates ranged in size between 10 and $35 \mathrm{~nm}$ and had a spherical shape. In vitro cellular uptake demonstrated that the TMAB-conjugated PAMAM was taken up by HER2-overexpressing BT474 cells more efficiently than MCF-7 cells that expressed lower levels of HER2. Co-localization experiments indicated that TMAB-conjugated PAMAM was located in the cytoplasm. The in vitro cytotoxicity of TMAB-conjugated PAMAM was lower than free PTX due to the slow release of PTX from the conjugate. In vivo targeting further demonstrated that TMAB-conjugated PAMAM accumulated in the BT474 tumor model more efficiently than non-conjugated PAMAM.

Conclusion: TMAB can serve as an effective targeting agent, and the TMAB-conjugated PAMAM can be exploited as a potential targeted chemotherapeutic drug delivery system for tumors that overexpress HER2.

Keywords: HER2, trastuzumab, PAMAM, active targeting, paclitaxel

\section{Introduction}

The ideal strategy in anticancer drug development includes targeted delivery to the tumor, low levels of adverse effects, and high efficacy. In recent years, nanocarrier systems have attracted increasing interest in this field. These targeted nanosystems can deliver drugs in either a passive or active manner. Passive targeted drug delivery utilizing the enhanced permeability and retention effect allows for increased deposition of nanovehicles at the site of a solid tumor. In contrast, active targeted drug delivery is achieved through covalent conjugation, on the nanocarrier surface, of a ligand or 
antibody that can identify and bind to a specific receptor that is overexpressed in cancer cells. ${ }^{1}$ Human epidermal growth factor receptor 2 (HER2), a member of the HER (also known as ErbB) family, is expressed weakly in normal adult tissues but overexpressed in 15\%-30\% of invasive breast cancers. ${ }^{2}$ The overexpression of HER2 on the tumor surface and the extracellular domain makes this protein an ideal target for active, targeted drug delivery. ${ }^{3}$

Trastuzumab (TMAB; brand name Herceptin ${ }^{\circledR}$ ) is a humanized monoclonal antibody that can specifically bind to the HER2 receptor. ${ }^{4}$ The conjugation of TMAB to nanoparticles enables the delivery of chemotherapeutic agents to specific tumor cells, thereby reducing adverse effects. TMAB has been used to target paclitaxel (PTX)-loaded poly(lacticco-glycolic acid) nanoparticles, ${ }^{5}$ poly(lactic-co-glycolic acid)/montmorillonite nanoparticles, ${ }^{6}$ and polyethylene glycol (PEG)ylated liposomes. ${ }^{7}$ These studies have shown promising results for the targeted delivery of PTX to cancer cells that overexpress HER2. ${ }^{5-7}$

Dendrimers are a family of nanosized, three-dimensional polymers characterized by a unique tree-like branching architecture. Polyamidoamine (PAMAM) dendrimers were the first commercialized dendrimers. They are synthesized using a small organic molecule, ethylenediamine, as the core, followed by stepwise addition of branches to the core. The unique advantages offered by PAMAM dendrimers over other drug delivery systems are their spherical shape, monodispersity, empty internal cavities, and many terminal surface groups $\left(\mathrm{NH}_{2}, \mathrm{OH}\right.$, and $\mathrm{COOH}$ surface groups). In addition, the surface groups can be easily modified with ligands to create dendrimers with broad applications. ${ }^{8}$ Due to their special chemical, physical, and biological features, PAMAM dendrimers have been widely used in drug delivery and have been increasing in popularity in recent years. ${ }^{9,10}$ Generally, drugs can be delivered by PAMAM through simple encapsulation, electrostatic interactions, and covalent conjugations. Covalent attachment of drugs to the surface groups via chemical bonds is more stable and offers better control over drug release compared to the encapsulation of a drug within hydrophobic cavities. ${ }^{11}$

In the present study, a PAMAM-PTX conjugate functionalized with anti-HER2 TMAB was developed, and the level of specific active targeting of the conjugate to the HER2 receptor both in vitro and in vivo was examined.

\section{Materials and methods Materials}

The PAMAM dendrimer (G4, 64 primary surface $\mathrm{NH}_{2}$ groups, $10 \% \mathrm{w} / \mathrm{w}$ solution in methanol), PTX, 2-iminothiolane
(Traut's reagent) and 5,5'-dithio-bis-(2-nitrobenzoic-acid) (Ellman's reagent) were purchased from Sigma-Aldrich Co (St Louis, MO, USA). $N$-hydroxysuccinimide-polyethylene glycol-maleimide (NHS-PEG-MAL, MW 3,500 Da) was supplied by JenKem Technology (Beijing, People's Republic of China). TMAB was purchased from Hoffman-La Roche Ltd (Basel, Switzerland). Sephadex G25 and HiPrep 16/60 Sephacryl S-300 HR were purchased from GE Healthcare Bio-Sciences Corp (Piscataway, NJ, USA). Fluorescein isothiocyanate (FITC), Hoechst 33342, and cyanine7 (Cy7)-NHS were purchased from Fanbo Biochemicals Co Ltd (Beijing, People's Republic of China). MTT (3-(4,5dimethylthiazol-2-Y1)-2,5-diphenyltetrazolium bromide), Dulbecco's Modified Eagle's Medium, and fetal bovine serum were purchased from Thermo Fisher Scientific (Waltham, MA, USA). Succinic anhydride, NHS, 4-dimethylaminopyridine (DMAP), and 1-(3-dimethylaminopropyl)3 -ethylcarbodiimide hydrochloride (EDC.HCI) were purchased from Aladdin Industrial Corp (Shanghai, People's Republic of China).

The breast cancer lines MCF-7 and BT474 and 4-week-old female nude mice were kindly provided by Luye Pharma Group (Shandong, People's Republic of China). All other materials were of reagent grade. All of the animal studies were performed according to the Guide for the Care and Use of Laboratory Animals. The China Animal Care and Use Committee approved the animal study protocols.

\section{Synthesis of TMAB-PEG-PAMAM- PTX \\ Synthesis of PTX-NHS ester}

PTX was conjugated to PAMAM through a succinic anhydride linker, as reported previously, with some modifications. ${ }^{12}$ Succinic anhydride $(17.6 \mathrm{mg}$ in $1 \mathrm{~mL}$ dichloromethane $[\mathrm{DCM}]$ ) was added dropwise to a stirred solution of $100 \mathrm{mg}$ PTX and $14.7 \mathrm{mg}$ DMAP in $6 \mathrm{~mL}$ DCM. The mixture was stirred for 6 hours at room temperature and then evaporated under vacuum to remove the DCM. The residue was dissolved in $1 \mathrm{~mL}$ DCM and purified on silica gel (washed with ethyl acetate) to yield $100 \mathrm{mg}$ PTX2'-hemisuccinate.

Approximately $1.15 \mathrm{~g}$ NHS and excess triethylamine were dissolved in $6 \mathrm{~mL} \mathrm{DCM}$ and stirred for 10 minutes, followed by the slow addition of $2.4 \mathrm{~g}$ diphenylphosphoryl chloride. The reaction solution was washed with $\mathrm{H}_{2} \mathrm{O}$, dried with $\mathrm{MgSO}_{4}$, and concentrated in a vacuum to produce $3.09 \mathrm{~g}$ N-hydroxysuccinimide diphenyl phosphate (SDPP). 
An appropriate amount of triethylamine was added to a stirred solution of $25.0 \mathrm{mg}$ PTX-2'-hemisuccinate and $12.4 \mathrm{mg}$ SDPP in $2 \mathrm{~mL}$ acetonitrile. The mixture was stirred for 6 hours at room temperature under $\mathrm{N}_{2}$ atmosphere, evaporated under vacuum, and purified on silica gel with a hexane/ ethyl acetate elution buffer $(90 / 10, \mathrm{v} / \mathrm{v})$ to produce $26.2 \mathrm{mg}$ PTX-NHS ester.

\section{Synthesis of PAMAM-PTX}

The PTX-NHS ester $(5.0 \mathrm{mg})$ and EDC $(5.1 \mathrm{mg})$ were dissolved in a $3 \mathrm{~mL}$ dimethylformamide and $1 \mathrm{~mL}$ dimethyl sulfoxide (DMSO) solvent mixture. This solution was stirred for 2 hours under $\mathrm{N}_{2}$ atmosphere and then added dropwise to a deionized water solution of PAMAM $(6.6 \mathrm{mg})$. The reaction mixture was stirred overnight at room temperature and then dialyzed (using a cellulose membrane with a 7,000 Da MW cutoff) against water for 24 hours. Filtration using a $0.2 \mu \mathrm{m}$ membrane and lyophilization yielded approximately $5.2 \mathrm{mg}$ of PAMAM-PTX.

\section{Synthesis of PEG-PAMAM-PTX}

The condensation reaction between PAMAM and PEG was carried out according to a previously reported method, with some minor modifications. ${ }^{13}$ PAMAM-PTX $(5 \mathrm{mg})$ was reacted with NHS-PEG-MAL at a ratio of 1:10 (mol/ $\mathrm{mol})$ in phosphate-buffered saline (PBS) $(\mathrm{pH} 8.0)$ for 2 hours at room temperature. The NHS group byproduct and excess NHS-PEG-MAL were removed by a Microcon ${ }^{\circledR}$ micro-concentrator (MW cutoff of [MWCO] 10,000 Da) until the cross-linker was eliminated from the filtrate, which was verified by thin layer chromatography using chloroform and methanol (10:1) as the mobile phase. The resulting product (PEG-PAMAM-PTX) was obtained via lyophilization, which yielded a white powder (10.9 mg, $85 \%$ yield).

\section{Synthesis of thiolated TMAB}

TMAB was thiolated using Traut's reagent, as previously described. ${ }^{14}$ The reaction conditions of TMAB thiolation were optimized. Briefly, TMAB (5 mg) was dissolved at a concentration of $10 \mathrm{mg} / \mathrm{mL}$ in $0.1 \mathrm{M}$ PBS (pH 8.0) containing $10 \mathrm{mM}$ ethylenediaminetetraacetic acid. Then, 2-iminothiolane was added to the TMAB solution to produce a molar excess of 20 , $50,75,100$, and 120 fold over the amount of the antibody, and the reaction was performed under $\mathrm{N}_{2}$ atmosphere at room temperature with gentle shaking for 0.5 hours. In addition, 2 iminothiolane was added to the TMAB solution to produce a molar excess of 50 fold over the amount of the antibody, and the reaction was performed for $0.25,0.5,1.0,2.0$ and
4.0 hours, respectively. Non-reacted 2-iminothiolane was removed using a Sephadex G25 gel column $(1 \mathrm{~cm} \times 30 \mathrm{~cm})$. Every $1 \mathrm{~mL}$ eluent fraction was collected and monitored for protein at $280 \mathrm{~nm}$. Fractions containing the thiolated antibodies were pooled and concentrated to $10 \mathrm{mg} / \mathrm{mL}$ using a Microcon micro-concentrator (MWCO 10,000 Da).

The number of thiol groups introduced to TMAB was determined using Ellman's reagent. Briefly, 6.25 $\mu \mathrm{L}$ Ellman's reagent was incubated with $250 \mu \mathrm{L}$ concentrated thiolated TMAB solution at room temperature. The absorbance at $412 \mathrm{~nm}$ was measured after 15 minutes, and the thiol concentration of the samples was determined relative to cysteine standard solutions, which were treated in the same manner.

\section{Synthesis of TMAB-PEG-PAMAM-PTX}

Thiolated TMAB in a volume of $0.5 \mathrm{~mL}$ (approximately $5.0 \mathrm{mg}$ ) was incubated with $1.86 \mathrm{mg}$ PEG-PAMAM-PTX $(1: 1 \mathrm{~mol} / \mathrm{mol})$ in PBS ( $\mathrm{pH} 7.4)$ at $4^{\circ} \mathrm{C}$ overnight under constant $\mathrm{N}_{2}$. TMAB-PEG-PAMAM-PTX was purified to remove PEG-PAMAM-PTX and free thiolated TMAB using gel filtration on a HiPrep 16/60 Sephacryl S-300 HR column $(1 \mathrm{~cm} \times 30 \mathrm{~cm})$. PBS was used as the mobile phase at a flow rate of $0.5 \mathrm{~mL} / \mathrm{min}$, and the elution was monitored at $280 \mathrm{~nm}$. The fractions containing the final product were pooled and concentrated using a Microcon micro-concentrator (MWCO $10,000 \mathrm{Da})$.

\section{Synthesis of TMAB-PEG-PAMAM- FITC/Cy 7 \\ Synthesis of PAMAM-FITC/Cy7}

FITC $(0.01 \mathrm{mmol})$ dissolved in $4 \mathrm{~mL}$ methanol was added to $10 \mathrm{~mL}$ PAMAM (0.002 mmol) methanol solution. The solution was stirred overnight in the dark at room temperature. After evaporation of the solvent, the residue was dissolved in $\mathrm{H}_{2} \mathrm{O}$ and dialyzed (MWCO at 7,000 Da) against $\mathrm{H}_{2} \mathrm{O}$ for 24 hours in darkness. The water was changed every 8 hours to remove the unreacted FITC. The absence of free FITC was verified by thin layer chromatography using chloroform and methanol (1:1) as the mobile phase. The obtained solution was concentrated using a Microcon micro-concentrator (MWCO 3,500 Da) and lyophilized to produce an orange powder $(29.2 \mathrm{mg})$.

Cy7-NHS (1 mg) dissolved in $0.1 \mathrm{~mL}$ DMSO was added to G4 PAMAM (3.48 mg) that was dissolved in $1 \mathrm{~mL}$ borate buffer ( $\mathrm{pH}$ 9.0). The solution was incubated for 4 hours in the dark at room temperature and then dialyzed (MWCO $7,000 \mathrm{Da}$ ) against water for 24 hours to remove unreacted Cy7-NHS. A green powder (4.44 mg) was obtained by lyophilization. 


\section{Synthesis of TMAB-PEG-PAMAM- FITC/Cy 7}

The synthesis procedure of TMAB-PEG-PAMAM-FITC/ Cy7 was similar to that of TMAB-PEG-PAMAM-PTX. Briefly, PAMAM-FITC/Cy7 was reacted with NHS-PEGMAL at the same ratio as TMAB-PEG-PAMAM-PTX and then conjugated to thiolated TMAB.

\section{Characterization of different PAMAM conjugates \\ Determination of the number of conjugated FITC/Cy7, PTX, PEG, and TMAB molecules}

The number of FITC/Cy7, PEG, and PTX molecules conjugated to G4 PAMAM was calculated by comparing the relative integral areas of the peaks originating from these components with PAMAM. The number of FITC/Cy7 moieties was also calculated relative to their standard solutions at $500 \mathrm{~nm}$ for FITC and $753 \mathrm{~nm}$ for Cy7. The amount of TMAB bound to the conjugate's surface was calculated using the Bradford Protein Assay Kit. In detail, $20 \mu$ L of TMAB-PEGPAMAM-PTX $(0.5 \mathrm{mg} / \mathrm{mL})$ was mixed with $20 \mu \mathrm{L}$ Brilliant Blue $\mathrm{G}$, incubated for 5 minutes at room temperature, and read on a spectrophotometer at $595 \mathrm{~nm}$. The concentration of TMAB was calculated relative to BSA standard solutions, which were treated in the same manner.

\section{Size and zeta potential}

The conjugates $(10 \mathrm{mg} / \mathrm{mL})$ were diluted with an appropriate volume of water, and the nanoparticle size, size distribution, and zeta potential were determined using a dynamic light scattering (DLS) particle size analyzer equipped with a $50 \mathrm{mV}$ laser at a scattering angle of $90^{\circ}$ (Nicomp $380 \mathrm{ZLS}$; PSS, Port Richey, FL, USA). The morphological examination of the conjugates was performed using a transmission electron microscope (TEM) (JEM 1400, JOEL, Japan) after staining with a $2 \%$ sodium phosphotungstate solution.

\section{In vitro targeting evaluation}

The qualitative and quantitative analyses of cellular uptake were performed using fluorescence microscopy (Eclipse E400; Nikon Corporation, Tokyo, Japan) and flow cytometry (BD FACSAria III, BD Biosciences, San Jose, CA, USA), respectively. The MCF-7 (low expression of HER2) and BT474 (overexpression of HER2) cells were seeded into 24-well plates with glass coverslips on the bottom at a density of 4,000 cells per well, and 6-well plates (for quantitative analysis) at a density of $1 \times 10^{4}$ cells per well. After the plates were incubated for 24 hours at $37^{\circ} \mathrm{C}$ in a $5 \% \mathrm{CO}_{2}$ atmosphere, the cells were treated with TMAB-PEG-PAMAM-FITC and PEG-PAMAM-FITC (as a control) for 1 hour in a series of concentrations ranging from $0.01 \mu \mathrm{M}$ to $1 \mu \mathrm{M}$ at $37^{\circ} \mathrm{C}$. The cells were washed three times with cold PBS and fixed with $4 \%$ paraformaldehyde for 15 minutes before visualization by fluorescence microscopy. For quantitative analyses, cells treated as described above were trypsinized and centrifuged at $1,500 \times g$ for 10 minutes. The cells were subsequently resuspended in PBS and analyzed by flow cytometry. Additionally, BT474 cells overexpressing HER2 were pre-incubated with $0.02 \mu \mathrm{M}$ TMAB for 30 minutes to block the receptor in order to confirm the specific binding of the TMAB-functionalized conjugate.

\section{Intracellular localization of conjugates}

BT474 cells were seeded into 24-well plates with glass coverslips in the bottom at a density of 4,000 cells per well and incubated for 24 hours at $37^{\circ} \mathrm{C}$ in a $5 \% \mathrm{CO}_{2}$ atmosphere. The cells were then treated with either TMAB-PEG-PAMAMFITC or PEG-PAMAM-FITC (as a control) for 1 hour at a concentration of $0.1 \mu \mathrm{M}$ at $37^{\circ} \mathrm{C}$. The cells were washed three times with cold PBS and fixed with $4 \%$ formaldehyde for 15 minutes. The endo-lysosomes were counterstained with $50 \mathrm{nM}$ LysoTracker Red for 30 minutes, and the nuclei were counterstained with $5 \mu \mathrm{g} / \mathrm{mL}$ Hoechst 33342 for 10 minutes. Confocal images were acquired using confocal microscopy (Olympus FV1000, Olympus Corporation, Tokyo, Japan).

\section{Evaluation of TMAB-PEG-PAMAM-PTX cytotoxicity}

The cytotoxicities of PTX, PAMAM-PTX, PEG-PAMAMPTX, and TMAB-PEG-PAMAM-PTX were determined using an MTT assay. BT474 cells in the logarithmic phase were removed from a culture flask by trypsinization and seeded in growth medium into a 96-well plate at a density of 2,000 cells per well. The plates were incubated for 24 hours at $37^{\circ} \mathrm{C}$ in a $5 \% \mathrm{CO}_{2}$ atmosphere. The cells were then treated with varying concentrations $\left(10^{-2}, 10^{-1}, 1,10,100,1,000\right.$, and $10,000 \mathrm{ng} /$ $\mathrm{mL}$ ) of different conjugate solutions for 24 hours at $37^{\circ} \mathrm{C}$. After the incubation, the cells were treated for 4 hours with $20 \mu \mathrm{L}$ MTT solution $(5 \mathrm{mg} / \mathrm{mL})$. The absorbance at $570 \mathrm{~nm}$ of each well was measured after gentle shaking for 10 minutes.

\section{In vivo targeting evaluation}

The whole-body distribution of the conjugate was determined using in vivo imaging (Care stream In Vivo FX, Bruker, 
Madison, WI USA) to demonstrate the targeting effect in vivo. TMAB-PEG-PAMAM-Cy7 (experimental group) and PEGPAMAM-Cy7 (control group) were injected intravenously into a nude mouse breast cancer model at an equivalent of $10 \mu \mathrm{g}$ Cy7. Images were captured at $0.5,1,2,4,8,12$, and 24 hours post-injection. The targeting efficiency was further confirmed using ex vivo imaging. The liver, spleen, lung, kidney, heart, and tumor tissue were harvested after the mice were euthanized with carbon dioxide at 24 hours post-injection. The mean fluorescence intensities of the tumor and normal tissues were recorded using the regions of interest function for semi-quantitative analysis.

\section{Data analysis}

The results are presented as the means \pm standard deviations (SD) of three samples. Statistical differences were determined using two-sample $t$-tests and analysis of variance, with $P<0.05$ defined as statistically significant.

\section{Results and discussion Synthesis of PEG-PAMAM-PTX}

The major disadvantage of most anticancer drugs is their high toxicity, which leads to adverse side effects. The treatment of breast cancer remains a problem due to lack of an effective drug delivery system. ${ }^{15}$ The goals of this study were to construct a TMAB-functionalized, breast cancer-targeting, highly efficient drug carrier, TMAB-PEG-PAMAM conjugate and to investigate the characteristics of this conjugate in vitro and in vivo. PTX was selected as a model drug to evaluate the specific anti-tumor effect of the targeted conjugate in vitro. The synthesis process is illustrated in Figure 1.

\section{PTX-NHS}

PTX was reacted with succinic anhydride to form an ester bond at the $\mathrm{C}^{\prime}$ ' position of PTX. The ester bond was used for conjugation because of its ease of enzymatic cleavage. ${ }^{12,16}$ A peak of PTX-2'-hemisuccinate was found at a molecular weight of $976.32 \mathrm{Da}\left(\left[\mathrm{M}^{+}+\mathrm{Na}\right]\right)$, which is consistent with its theoretical molecular weight (976.99 g/mol, Figure S1). The reaction speed was greatly accelerated compared to a previous method ${ }^{17}$ through the application of DMAP as a catalyst. PTX-2'-hemisuccinate was coupled with SDPP (Figure S2) to synthesize an activated PTX-NHS ester. The use of SDPP gave better yields and milder reaction conditions than did any of the carbodiimide coupling reagents. A peak for the PTXNHS ester was found at a molecular weight of 1,073.35 Da $\left(\left[\mathrm{M}^{+}+\mathrm{Na}\right]\right)$, which is consistent with the theoretical molecular weight $(1,074.08 \mathrm{~g} / \mathrm{mol}$, Figure S3). The synthesis process is illustrated in Figure S4.

\section{PAMAM-PTX}

The successful synthesis of PAMAM-PTX was confirmed using proton nuclear magnetic resonance $\left({ }^{1} \mathrm{H}-\mathrm{NMR}\right)$, which showed both PTX and PAMAM peaks (Figure S5). The peaks at $\delta(\mathrm{ppm}) \approx 7.0-8.0$ belong to the three phenyl groups (15 H's) of PTX. The peaks at $\delta(\mathrm{ppm}) \approx 2.2-3.6$ belong to the methylene protons of the branching units of PAMAM, which is consistent with the previously reported ${ }^{1} \mathrm{H}-\mathrm{NMR}$ of PAMAM. ${ }^{18}$ The number of PTX molecules that conjugated to PAMAM was calculated using the proton integration method by taking the characteristic peaks of PTX and PAMAM dendrimer into account, as previously described. ${ }^{19}$ The result indicated that approximately four PTX molecules were attached to a PAMAM dendrimer.

\section{PEG-PAMAM-PTX}

A hetero-bifunctional cross-linker with NHS ester and maleimide groups was reacted with the primary amino groups on the surface of PAMAM. The modification of PAMAM with NHS-PEG-MAL can produce an intermediate that is coated with PEG spacers. This intermediate can be used to promote PAMAM hydrophilicity, avoid non-specific interactions, decrease cytotoxicity, ${ }^{20}$ and provide thiol-reactive maleimides for coupling thiolated TMAB. NHS-PEG-MAL conjugation to G4 PAMAM was confirmed by the appearance of a signal at $3.6 \mathrm{ppm}$ that corresponded to the methylene protons of PEG (Figure 2). The number of PEG molecules that conjugated to PAMAM was calculated as eight molecules using the proton integration method.

\section{Synthesis of PEG-PAMAM-FITC/Cy7}

Fluorescent probes were used both in the in vitro imaging of cells via fluorescence microscopy, flow cytometry, or confocal microscopy, and the in vivo imaging of nude mice via Care stream In Vivo FX. The absorbance at $\lambda_{\text {max }}$ $500 \mathrm{~nm}$ for FITC and $\lambda_{\max } 753 \mathrm{~nm}$ for Cy 7 initially indicated the successful conjugation of the fluorescent probes. ${ }^{21}$ The ${ }^{1} \mathrm{H}-\mathrm{NMR}$ spectra showed aromatic proton peaks for FITC and $\mathrm{Cy} 7$ from 6.5 to $8.0 \mathrm{ppm}$, whereas those of the PAMAM methylenes were observed from 2.0 to $3.6 \mathrm{ppm}$ (Figure 3). The ${ }^{1} \mathrm{H}-\mathrm{NMR}$ results confirmed that FITC and $\mathrm{Cy} 7$ were successfully conjugated to PAMAM. The number of conjugated FITC molecules per dendrimer was calculated at four molecules using the proton integration method. This result was further confirmed by ultraviolet-visible spectroscopy using a FITC concentration calibration curve. The number of conjugated $\mathrm{Cy} 7$ molecules per dendrimer was calculated at one molecule using the same methods. The degree of PEG 


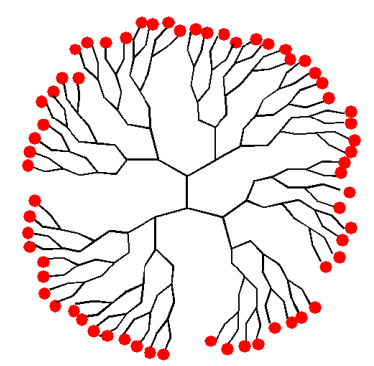

(1) FITC

methanol, overnight RT

(2) Cy7-NHS,

DMSO/borate buffer ( $\mathrm{pH}$ 9.0)

4 h RT

(3) PTX-NHS

DMSO/DMF/ $\mathrm{H}_{2} \mathrm{O}$

overnight RT
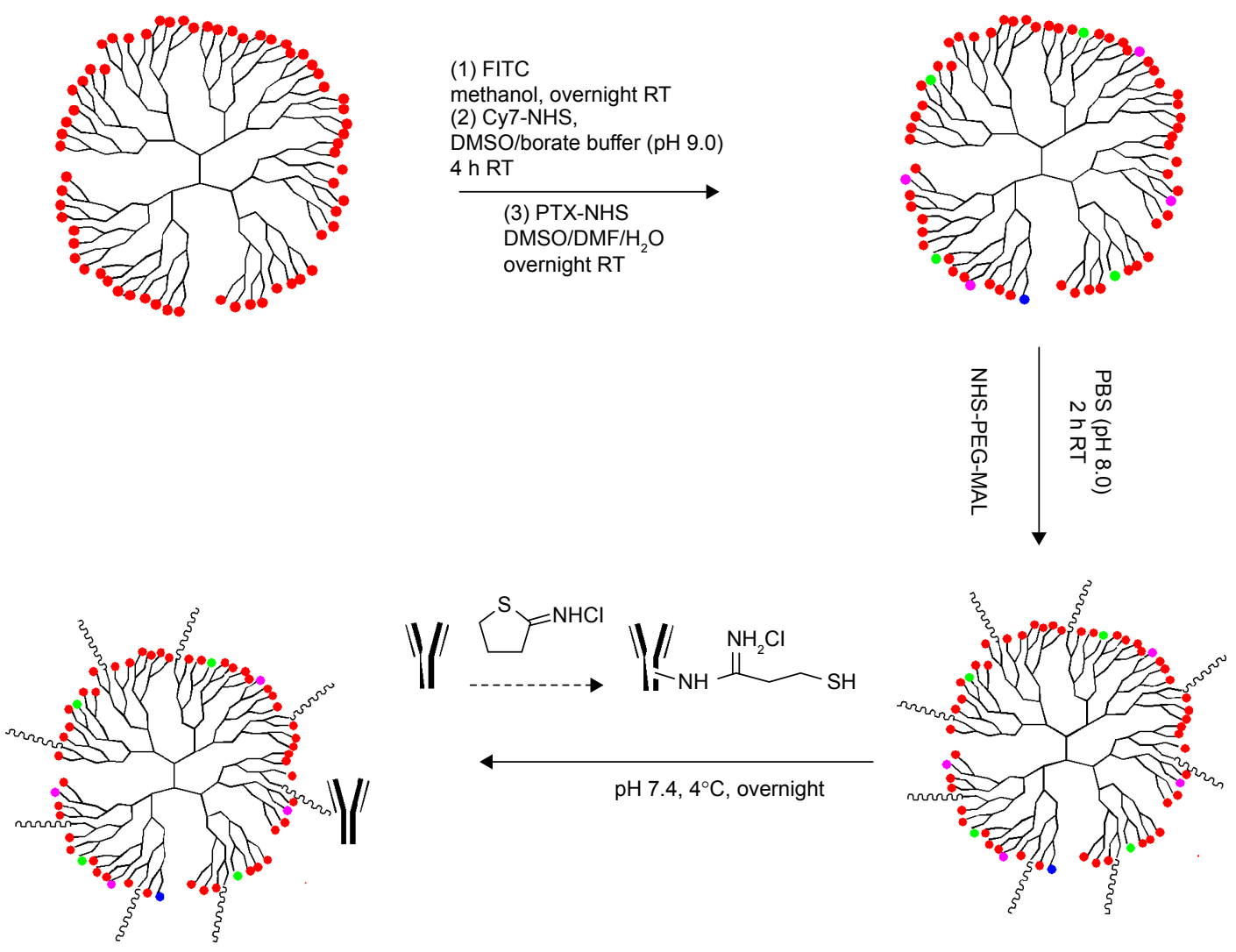

$\bullet=\mathrm{NH}_{2}$

$\bullet=$ FITC

$\bullet=\mathrm{Cy} 7-\mathrm{NHS}$

$\bullet=$ PTX-NHS

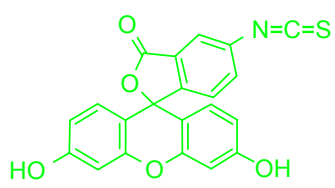

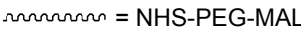

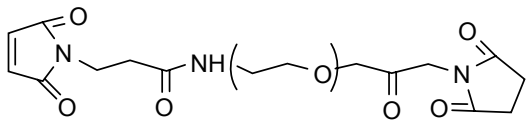

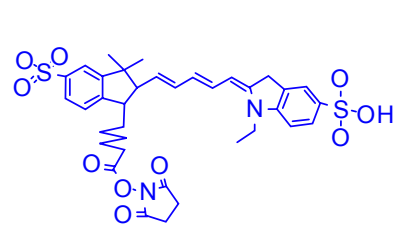

$\mathrm{O}=1$

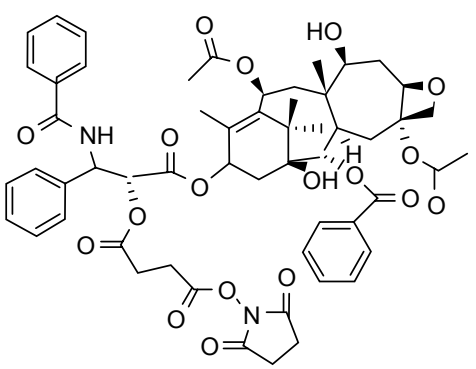

$\|=$ =

Figure I Schematic synthesis of TMAB-functionalized conjugates.

Abbreviations: TMAB, trastuzumab; PTX-NHS, paclitaxel-N-hydroxysuccinimide; NHS, N-hydroxysuccinimide; PEG, polyethylene glycol; MAL, maleimide; FITC, fluorescein isothiocyanate; Cy7, cyanine7; PTX, paclitaxel; PBS, phosphate-buffered saline; DMSO, dimethyl sulfoxide; DMF, dimethylformamide; h, hours; RT, room temperature.

modification of these two conjugates (12.5\%) was made the same as with PEG-PAMAM-PTX by controlling the feed ratio of the starting materials.

\section{Synthesis of TMAB-PEG-PAMAM-PTXI FITC/Cy 7}

One critical step in the synthesis of targeted conjugates was the thiolation of TMAB, because TMAB was covalently attached via its thiolated form to a sulfhydryl reactive group introduced by a bi-functional PEG cross-linker onto the surface of PAMAM. Figure 4 shows that the number of sulfhydryls did not increase with reaction time. TMAB thiolation reached a maximum after 1 hour, and then it slightly decreased. Because antibodies are easily deactivated, 1 hour was selected as the reaction time. No significant differences were found in the degree of thiolation when the molar ratio of 


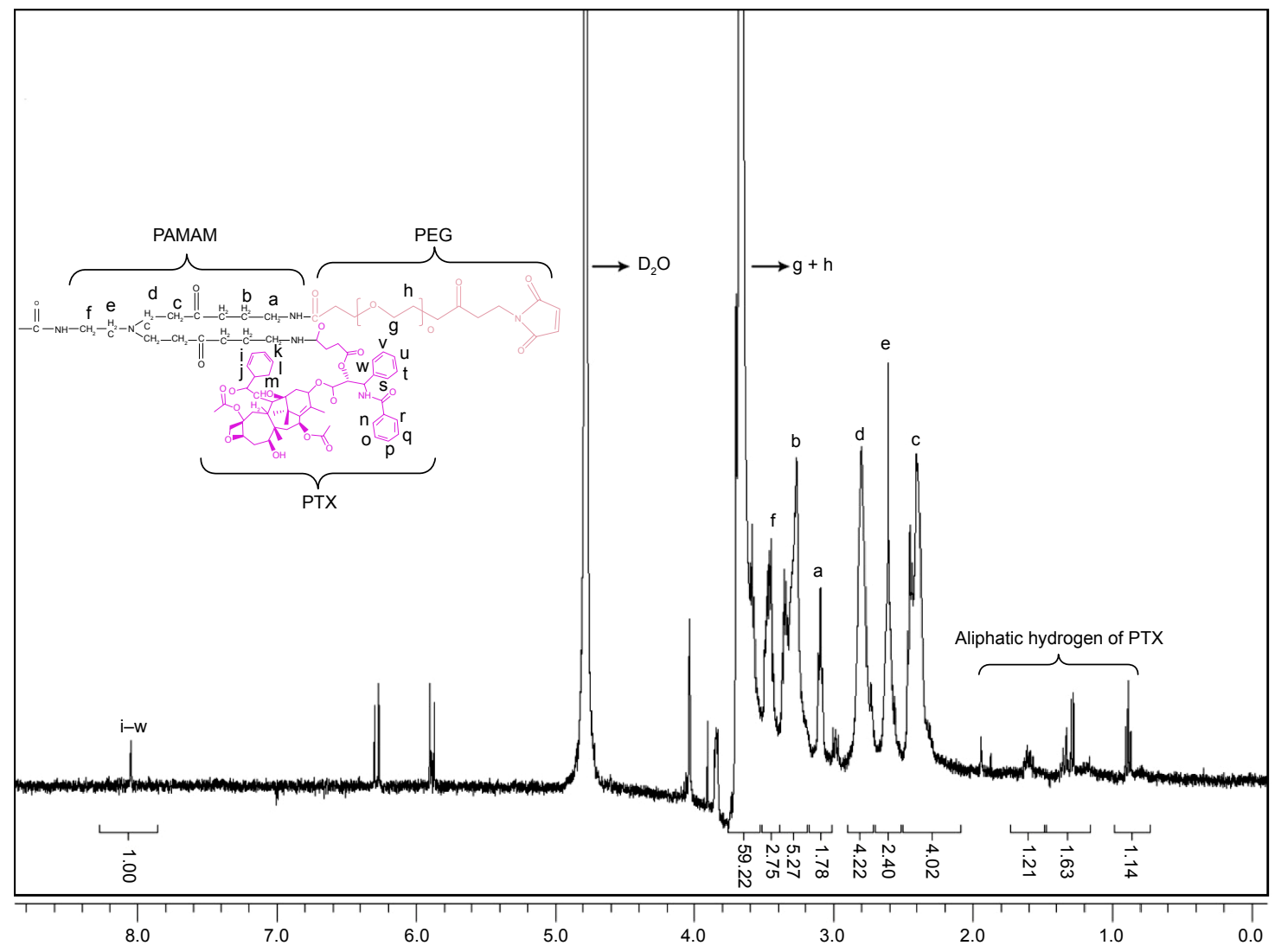

Figure 2 Proton nuclear magnetic resonance ('H-NMR) spectra of PEG-PAMAM-PTX in $\mathrm{D}_{2} \mathrm{O}$ at $400 \mathrm{MHz}$. Note: $a-w$ represent the protons of PEG-PAMAM-PTX.

Abbreviations: PEG, polyethylene glycol; PAMAM, polyamidoamine; PTX, paclitaxel.

Traut's reagent to TMAB was 10:1 or greater. Because thiolated TMAB could be purified from excess Traut's reagent by gel filtration according to the elution curve (Figure S6), a molar ratio of 20 was selected as the reaction ratio.

As shown in Figure 5, the absorbance of TMAB-PEGPAMAM-FITC/Cy7 at $280 \mathrm{~nm}$ was increased compared to that of PAMAM-FITC/Cy7, which preliminarily demonstrated that TMAB was conjugated to PEG-PAMAM. Furthermore, sodium dodecyl sulfate polyacrylamide gel electrophoresis was also used to confirm the conjugation; as shown in Figure 6, bands of TMAB-functionalized conjugate were above the TMAB band, which confirmed the successful conjugation. The fluorescence of the conjugates provided indirect proof of successful conjugation, because the fluorescent probes were conjugated to the PAMAM dendrimer. ${ }^{22}$ The Bradford method was selected to calculate the amount of TMAB instead of the UV method because of interference from FITC and Cy7 at $280 \mathrm{~nm} .{ }^{23}$ The number of conjugated TMAB molecules was calculated as $1 \pm 0.02$ molecules using the Bradford method. There were eight PEG molecules conjugated to PAMAM; thus, there should be eight maleimide groups available to react with thiolated TMAB. However, only one TMAB molecule was conjugated to PEG. Our previous study attempted to conjugate additional TMAB moieties to enhance the conjugate's targeting ability, but these efforts failed, which might have been caused by the steric hindrance and electrostatic interaction between TMAB and PAMAM. Therefore, TMAB was reacted with PEG-PAMAM at a ratio of $1: 1$ to minimize the amount of free TMAB.

\section{Morphology, size, and zeta potential}

We investigated the physical characteristics of the different conjugates with regard to mean diameter, polydispersity index, and zeta potential. Table 1 shows the mean diameter and zeta potential of the different conjugates, which were determined using DLS. The particles with a size of $<5 \mathrm{~nm}$ would be rapidly eliminated by the kidney, and the leakiness of the particles to the nascent vasculature of tumors would be inhibited at particle sizes $>100 \mathrm{~nm} .{ }^{24}$ In the present study, three different conjugates (TMAB-PEG-PAMAMFITC/Cy7/PTX) had an average particle size of $25 \pm 10.5$, $34 \pm 12.3$, and $35.8 \pm 11.8 \mathrm{~nm}$, respectively. The mean diameter 

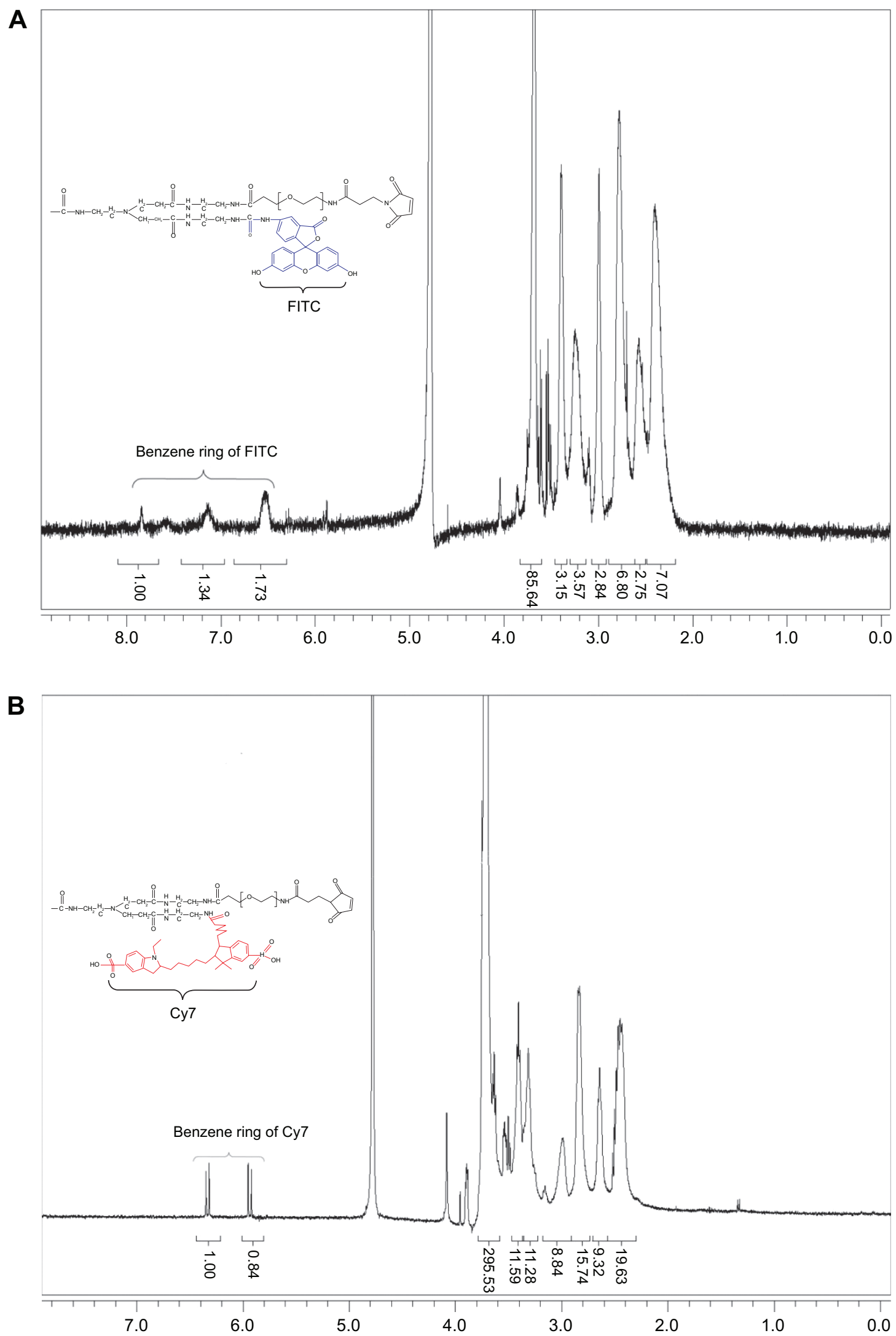

Figure 3 Proton nuclear magnetic resonance ('H-NMR) spectra of (A) PEG-PAMAM-FITC and (B) PEG-PAMAM-Cy7 in D 20 at 400 MHz. Abbreviations: PEG, polyethylene glycol; PAMAM, polyamidoamine; FITC, fluorescein isothiocyanate; Cy7, cyanine7. 

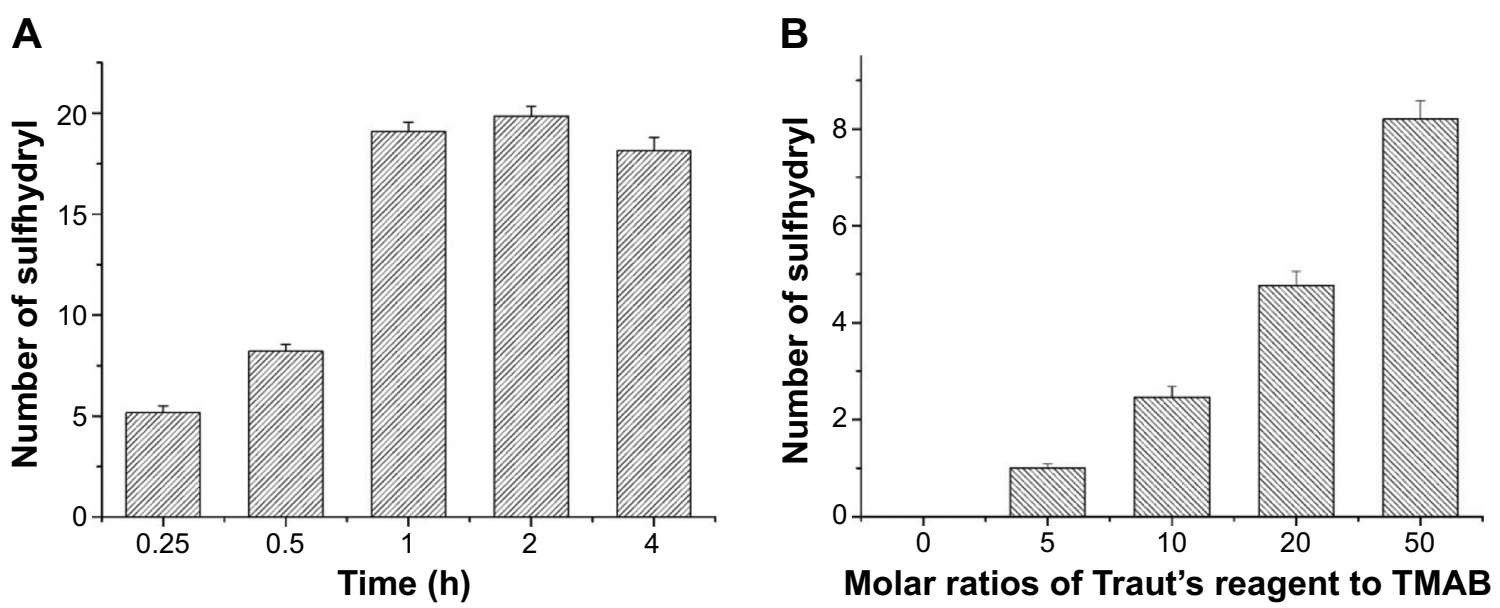

Figure 4 (A) The effect of different thiolation times on the number of sulfhydryls.

(B) The effect of different molar ratios of Traut's reagent to TMAB on the number of sulfhydryls.

Note: The data represent the mean \pm SD of three independent experiments.

Abbreviations: TMAB, trastuzumab; SD, standard deviation; $h$, hours.

of the TMAB-functionalized conjugates increased as a result of the attachment of TMAB. However, its diameter remained $<100 \mathrm{~nm}$, which is necessary to avoid uptake by the reticuloendothelial system. ${ }^{25}$ The zeta potential decreased after conjugation with TMAB, which could have been caused by the negative charge of TMAB. As shown in Figure 7, the morphology of the different conjugates visualized by negative stain TEM were approximately round, and the average particle sizes of three different conjugates (TMAB-PEGPAMAM-FITC/Cy7/PTX) were 20.8, 15.4, and $21.3 \mathrm{~nm}$, respectively. The particle sizes visualized by TEM were smaller than those determined by DLS. This result could be explained by the fact that the particle size determined by DLS was the hydrodynamic size, whereas TEM determined the dry sample size. The stretching of the PEG chains and the hydration layer will increase the particle size.${ }^{26}$ DLS measurement
A

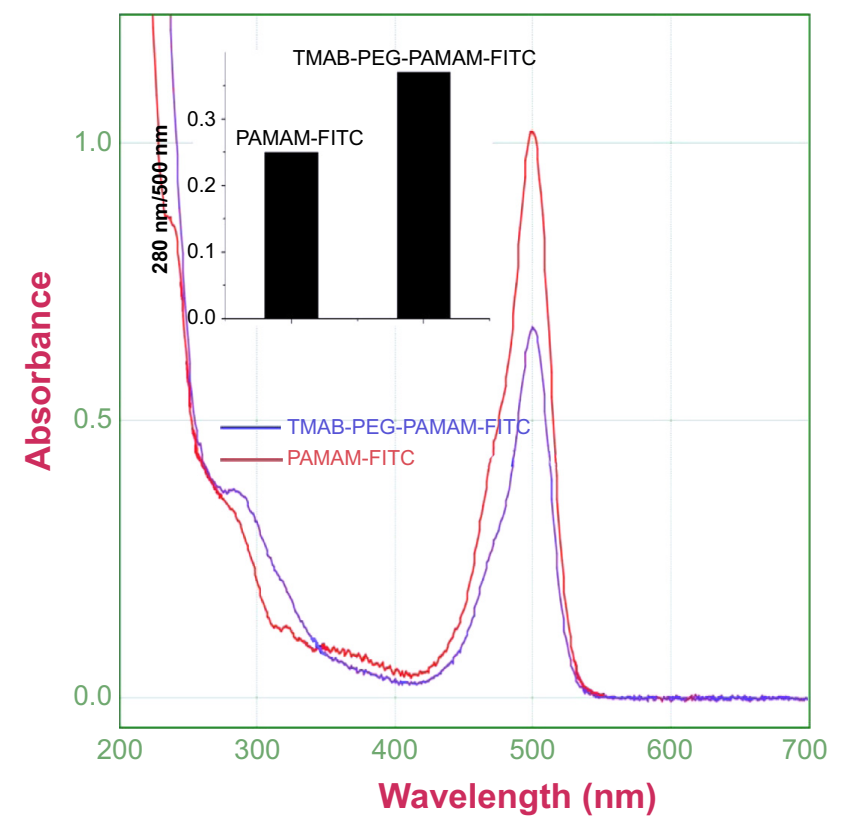

B

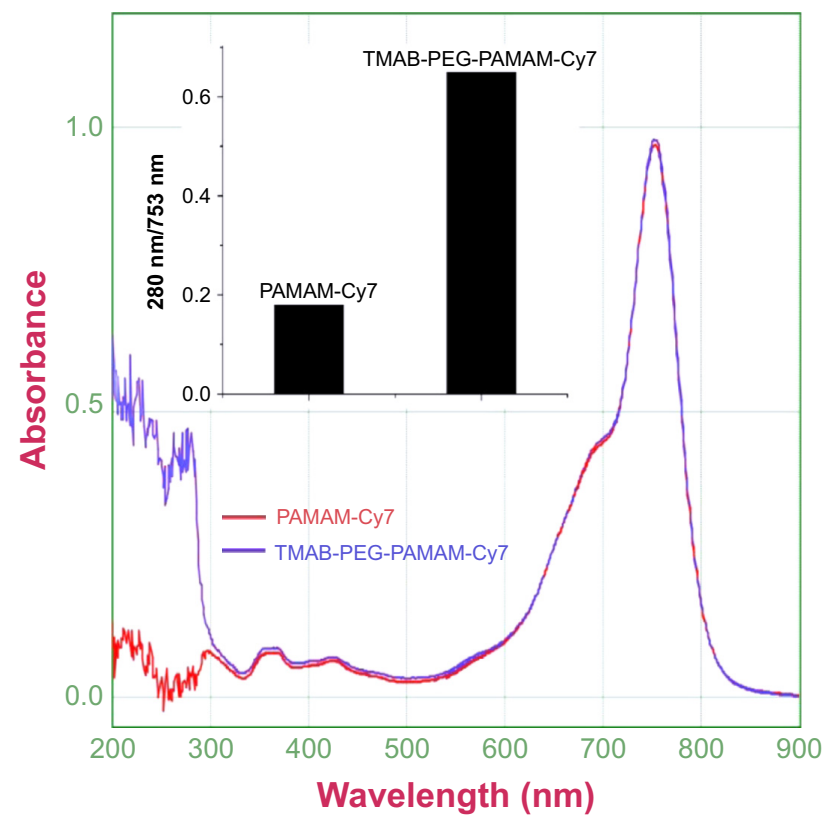

Figure 5 UV-Vis spectroscopy of PAMAM-FITC, TMAB-PEG-PAMAM-FITC, PAMAM-Cy7, TMAB-PEG-PAMAM-Cy7 in $\mathrm{H}_{2} \mathrm{O}$.

Note: The increased 280/500 (750) nm ratio (A) for the TMAB-PEG-PAMAM-FITC (Cy7) (B) vs PAMAM-FITC (Cy7) due to the conjugation of TMAB in the former. Abbreviations: UV-Vis, ultraviolet-visible; TMAB, trastuzumab; FITC, fluorescein isothiocyanate; PAMAM, polyamidoamine; vs, versus; PEG, polyethylene glycol; Cy7, cyanine7. 
Table I Size and zeta potential of different conjugates

\begin{tabular}{llll}
\hline & Particle size $(\mathbf{n m})$ & Polydispersity & Zeta potential $(\mathbf{m V})$ \\
\hline PAMAM-FITC & $11.5 \pm 7.2$ & 0.393 & $7.35 \pm 2.1$ \\
PEG-PAMAM-FITC & $17 \pm 9.8$ & 0.333 & $0.35 \pm 0.12$ \\
TMAB-PEG-PAMAM-FITC & $25 \pm 10.5$ & 0.178 & $-3.56 \pm 0.23$ \\
PAMAM-Cy7 & $13.3 \pm 6.7$ & 0.255 & $4.23 \pm 0.15$ \\
PEG-PAMAM-Cy7 & $24.2 \pm 10.2$ & 0.178 & $0.18 \pm 0.09$ \\
TMAB-PEG-PAMAM-Cy7 & $34 \pm 12.3$ & 0.130 & $-3.86 \pm 1.22$ \\
PAMAM-PTX & $15.1 \pm 7.0$ & 0.219 & $2.47 \pm 0.07$ \\
PEG-PAMAM-PTX & $30.8 \pm 11.5$ & 0.139 & $0.90 \pm 0.04$ \\
TMAB-PEG-PAMAM-PTX & $35.8 \pm 11.8$ & 0.108 & $-0.47 \pm 0.11$ \\
\hline
\end{tabular}

Abbreviations: PAMAM, polyamidoamine; FITC, fluorescein isothiocyanate; PEG, polyethylene glycol; TMAB, trastuzumab; Cy7, cyanine7; PTX, paclitaxel.

is sensitive to the size of whole particles. Bigger particles will contribute more to the average particle size. TEM measurements reflect the real particle size. However, TEM is limited by the number of particles in the field of vision. ${ }^{27}$

\section{In vitro targeting evaluation}

The cellular uptake of TMAB-PEG-PAMAM-FITC was measured in MCF-7 (low HER2 expression) and BT474 (high HER2 expression) cells by flow cytometry. Figure 8 showed that the mean fluorescence intensities of MCF-7 (9 \pm 3 to $110 \pm 12)$ and BT474 (10 \pm 3 to $327 \pm 25)$ cells increased after incubation with increasing concentrations of TMAB-PEGPAMAM-FITC $(0.05-1 \mu \mathrm{M})$ for 1 hour. This result indicated that the cellular uptake of TMAB-PEG-PAMAM-FITC by MCF-7 and BT474 cells was concentration-dependent. At a fixed concentration, TMAB-PEG-PAMAM-FITC was taken

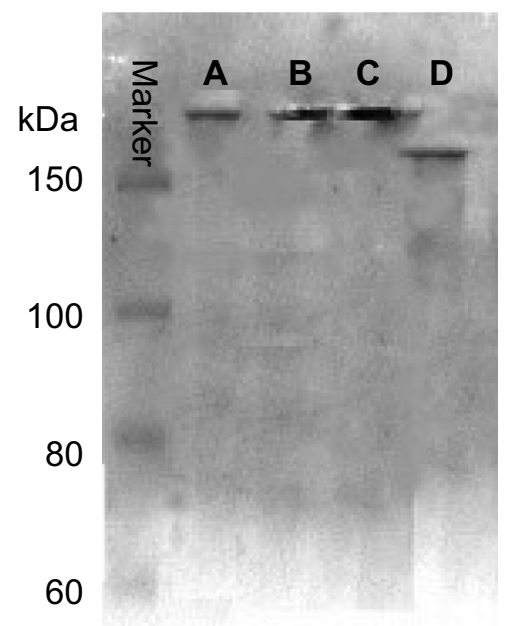

Figure 6 Non-reducing SDS-PAGE of different conjugates. Lanes A-D are TMABPEG-PAMAM-PTX, TMAB-PEG-PAMAM-FITC, TMAB-PEG-PAMAM-Cy7, and TMAB, respectively.

Abbreviations: SDS-PAGE, sodium dodecyl sulfate polyacrylamide gel electrophoresis; TMAB, trastuzumab; PEG, polyethylene glycol; PAMAM, polyamidoamine; PTX, paclitaxel; FITC, fluorescein isothiocyanate; Cy7, cyanine7. up by BT474 cells more efficiently than by MCF-7 cells, which suggested that TMAB conjugation increased cellular uptake by the HER2-overexpressing BT474 cells. This result was consistent with the fluorescence microscopy images. Because the surfaces of the BT474 cells had high expression levels of HER2, TMAB could facilitate uptake of the conjugate more efficiently. In addition, PEG-PAMAM-FITC showed similar cellular uptake by MCF-7 and BT474 cells. The cellular uptake by MCF-7 compared with TMAB-PEGPAMAM-FITC was not significantly different, whereas the cellular uptake by BT474 was significantly lower than that of TMAB-PEG-PAMAM-FITC.

To confirm the receptor specificity for the TMABfunctionalized conjugate, the effect of free TMAB on the uptake of the conjugate was studied by pretreating the cells with free TMAB to block the cell surface receptors. As shown in Figure 9, the cellular uptake of TMAB-PEGPAMAM-FITC was greatly inhibited by pretreatment with free TMAB (the percentage of FITC positive cells decreased from $65.32 \%$ to $0.79 \%$ ), but PEG-PAMAM-FITC exhibited no significant differences (the percentage of FITC positive cells decreased from $51.26 \%$ to $43.52 \%$ ). Binding and internalization properties of the conjugates were further confirmed by confocal microscopy. The fluorescence of TMAB-PEG-PAMAM-FITC decreased more than that of PEG-PAMAM-FITC (Figure 9A-D). The first step in the cellular uptake of a drug delivery system is its interaction with the cell membrane, including non-specific interactions, such as electrostatic interactions, and specific interactions, such as receptor-ligand binding. ${ }^{28}$ PEG-PAMAM-FITC has a positive charge (zeta potential $=0.35 \mathrm{mV}$ ) and could interact with the negatively charged cell membrane. However, TMAB-PEG-PAMAM-FITC has a negative charge (zeta potential $=-3.56 \mathrm{mV}$ ) and could not interact with the cell membrane through electrostatic interaction. Thereafter, 
A

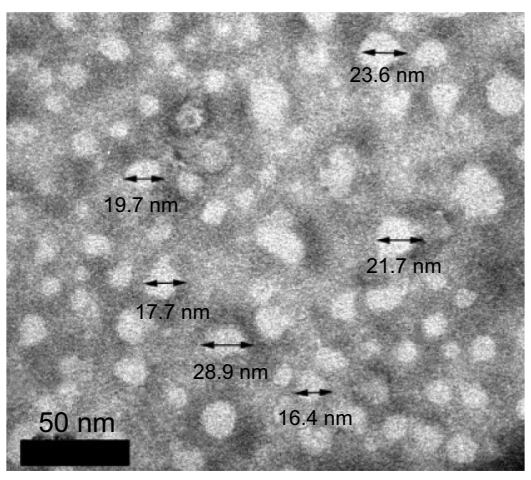

B

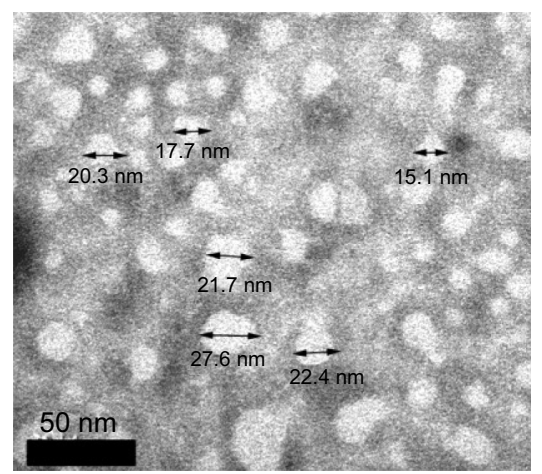

C

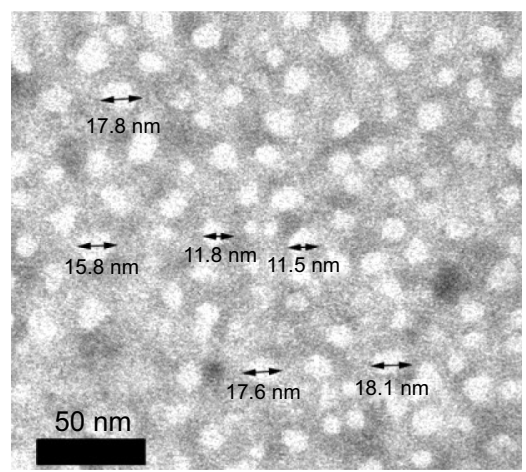

Figure 7 Transmission electron microscopy images of (A) TMAB-PEG-PAMAM-PTX, (B) TMAB-PEG-PAMAM-FITC, and (C) TMAB-PEG-PAMAM-Cy7. Abbreviations: TMAB, trastuzumab; PEG, polyethylene glycol; PAMAM, polyamidoamine; PTX, paclitaxel; FITC, fluorescein isothiocyanate; Cy7, cyanine7.

it could be inferred that the cellular uptake of TMABPEG-PAMAM-FITC in cells overexpressing HER2 was receptor-mediated, whereas the cellular uptake of PEGPAMAM-FITC was due to electrostatic interactions.

The intracellular localization of conjugates was performed in BT474 cells and visualized by confocal microscopy. As shown in Figure 10, TMAB-PEG-PAMAM-FITC co-localized with the lysosomes and was observed surrounding (but not co-localized with) the nuclei. The majority of TMABPEG-PAMAM-FITC conjugates co-localize in the lysosome. These results indicated that TMAB-PEG-PAMAM-FITC was endocytosed by BT474 cells and targeted to lysosomes. In addition, PEG-PAMAM-FITC exhibited a similar result, which is consistent with a previous report. ${ }^{29}$

\section{Evaluation of TMAB-PEG-PAMAM-PTX cytotoxicity}

The cytotoxicity of different conjugates against BT474 cells was tested. As shown in Figure 11, the half maximal inhibitory concentration $\left(\mathrm{IC}_{50}\right)$ value of TMAB-PEG-PAMAM-PTX was higher than that of free drug, indicating a lower toxicity of TMAB-PEG-PAMAM-PTX. As this conjugate was located in
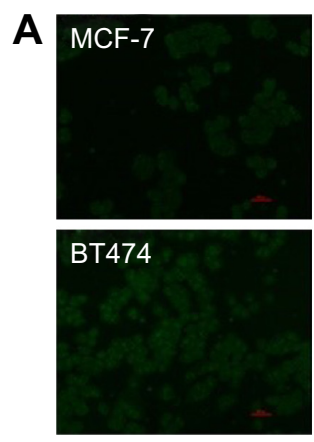

B

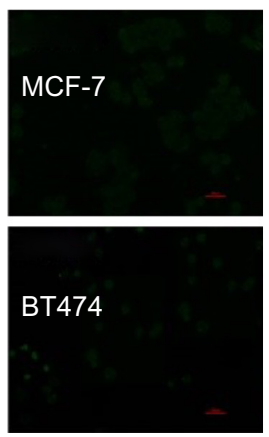

0.05
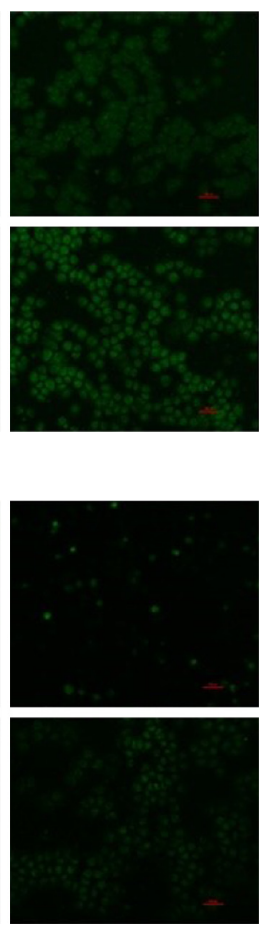

0.1
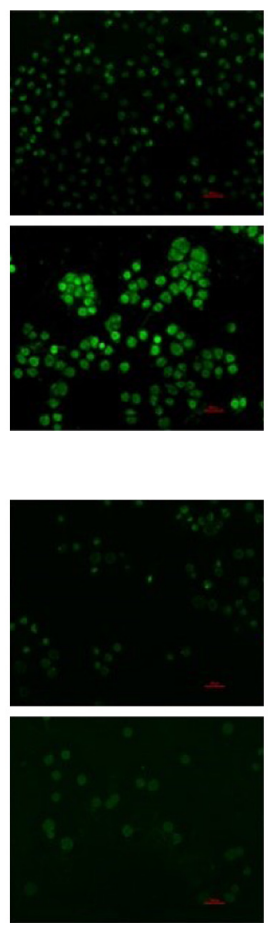

0.25
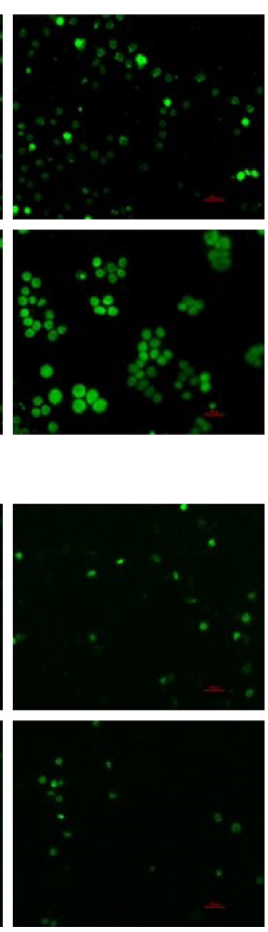

0.5
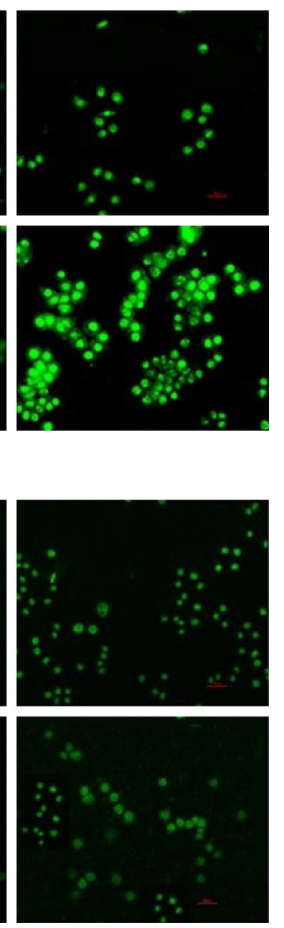

1

Figure 8 (Continued) 


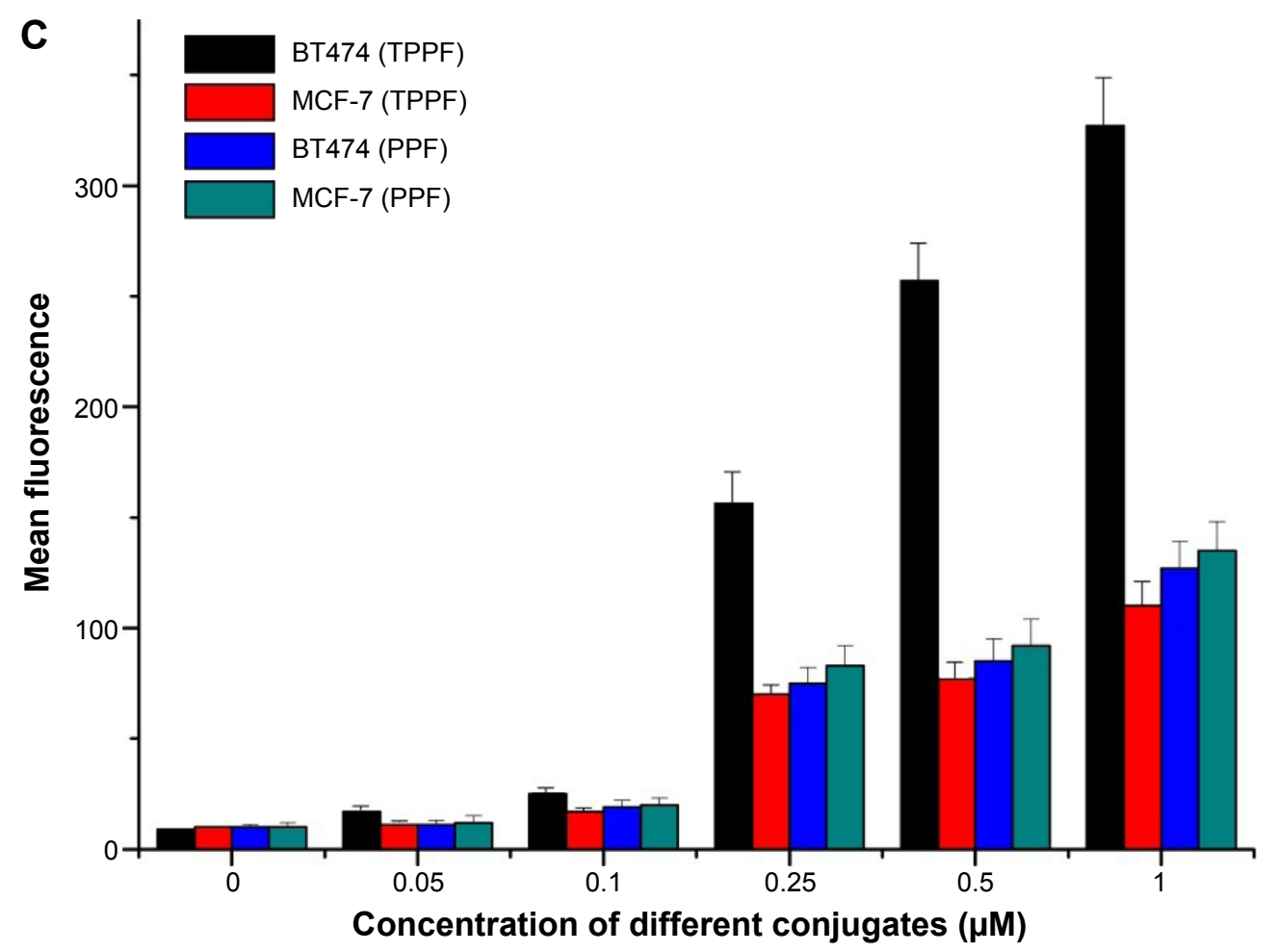

Figure 8 Concentration-dependent binding of TMAB-PEG-PAMAM-FITC and PEG-PAMAM-FITC to BT474 cells (overexpression of HER2) and MCF-7 cells (low expression of HER2). (A) Fluorescence microscopy images of TMAB-PEG-PAMAM-FITC; (B) fluorescence microscopy images of PEG-PAMAM-FITC; and (C) mean fluorescence as measured by flow cytometry.

Notes: The values are the mean \pm SD of three independent experiments. The scale bar for $A$ and $B$ is $50 \mu \mathrm{m}$.

Abbreviations: TMAB, trastuzumab; PEG, polyethylene glycol; PAMAM, polyamidoamine; FITC, fluorescein isothiocyanate; HER2, human epidermal growth factor receptor 2; SD, standard deviation; TPPF, TMAB-PEG-PAMAM-FITC; PPF, PEG-PAMAM-FITC.

the cytoplasm and would have an effect after PTX was released into the cytoplasm, the cytotoxicity was likely decreased due to the slow release of the drug from the conjugate within a set period of time. However, the increased cytotoxicity of free PTX in vivo causes adverse side effects because of its nonselective distribution in normal tissue. ${ }^{30} \mathrm{~A}$ previous report also found that the cytotoxicity of targeted scFv-425-SNAP-PGDoxo-PEG (a dendritic, polyglycerol based multifunctional doxorubicin immunoconjugate that specifically targets and kills cancer cell lines expressing epidermal growth factor receptor), was lower compared to that of the control doxorubicin, but the specificity was higher. ${ }^{31}$ It should be noted that the $\mathrm{IC}_{50}$ value of TMAB-PEG-PAMAM-PTX was lower than that of PEG-PAMAM-PTX due to the receptor-mediated specific cellular uptake of TMAB-PEG-PAMAM-PTX by BT474 cells. These results suggest that the TMAB-modified PAMAM dendrimer can be used to specifically deliver anticancer drugs to cells overexpressing HER2.

\section{In vivo targeting of TMAB-PEG- PAMAM-Cy7}

Cy7-NHS, an amine reactive dye in the near infrared spectrum, is highly hydrophilic. Near infrared fluorophores can be used to take advantage of the near-infrared window of biological tissues. Highly increased transparency of tissues in this spectral region allows for in vivo imaging. ${ }^{32}$ In the study, Cy7-NHS was conjugated to PAMAM to monitor the biodistribution of different conjugates in BT474 tumor-bearing nude mice. ${ }^{33}$ Figure 12 shows the in vivo fluorescence images of the BT474 tumor-bearing nude mice at predefined times after tail vein injections of either TMAB-PEG-PAMAM-Cy7 or PEG-PAMAM-Cy7. A weak signal was observed in the tumor tissue 2 hours after the TMAB-PEG-PAMAM-Cy7 injections. The signal increased gradually thereafter, reaching a maximum value at 12 hours post-injection, and was still detectable in the tumors at 24 hours. Most importantly, the mice in the TMAB-PEG-PAMAM-Cy7 group showed a stronger signal in the tumor compared to the mice in the PEG-PAMAM-Cy7 group at most time points. This result provided convincing evidence of the active targeting effect of the TMAB-modified PAMAM dendrimer.

Images of the excised tissues were obtained after 24 hours to further confirm the distribution of the conjugates. The strongest signal in the test group was observed in tumor tissues, followed by the spleen and liver tissues. In contrast, the control group showed the strongest signal in the 

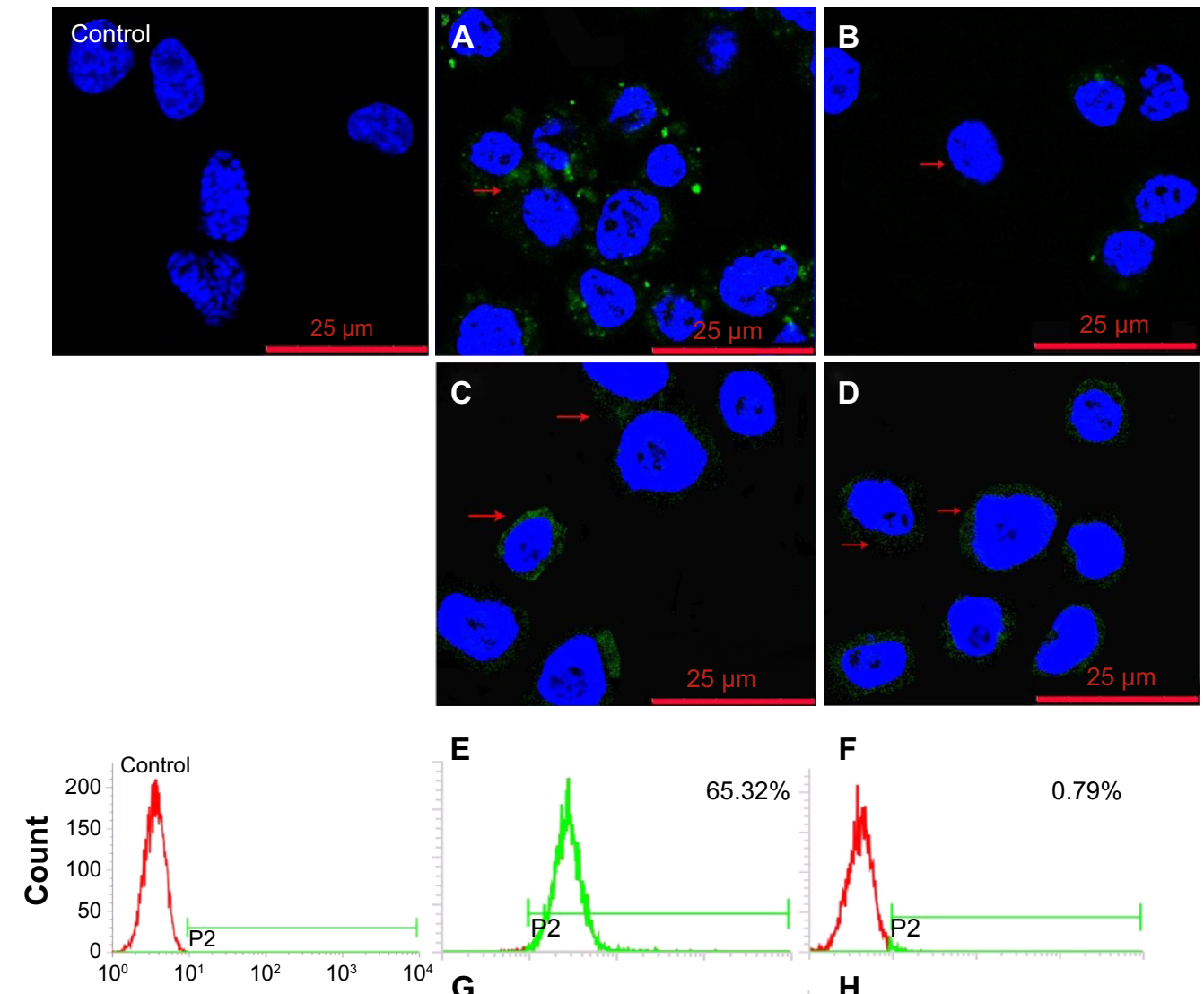

E

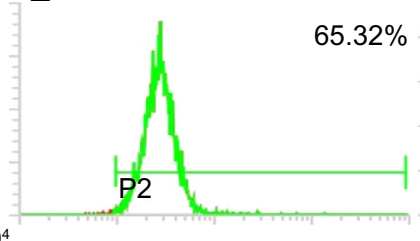

$\mathbf{F}$
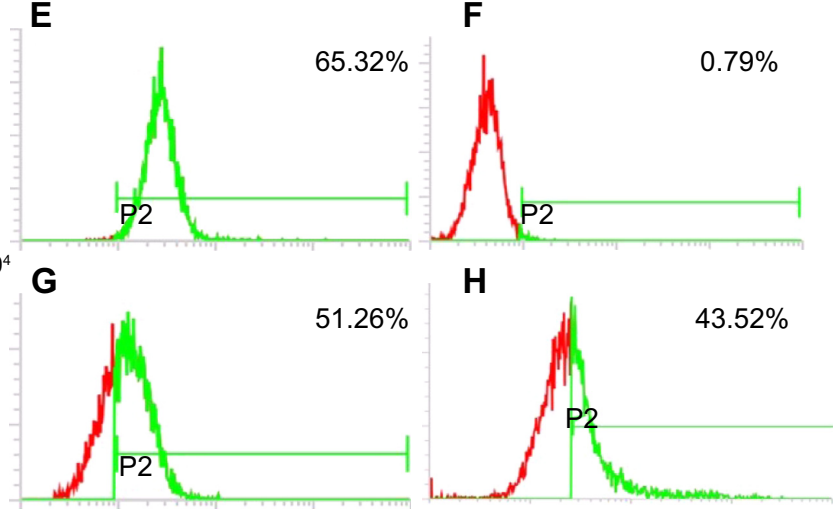

Figure 9 Confocal fluorescence microscopy images and flow cytometry analysis of TMAB-PEG-PAMAM-FITC (A, B, E, F) and PEG-PAMAM-FITC (C, D, G, H) binding to BT474 cells pretreated with TMAB (0.2-fold molar excess) for $30 \mathrm{~min}$. The red arrows indicate fluorescence of TMAB-PEG-PAMAM-FITC and PEG-PAMAM-FITC. Abbreviations: TMAB, trastuzumab; PEG, polyethylene glycol; PAMAM, polyamidoamine; FITC, fluorescein isothiocyanate; min, minutes.
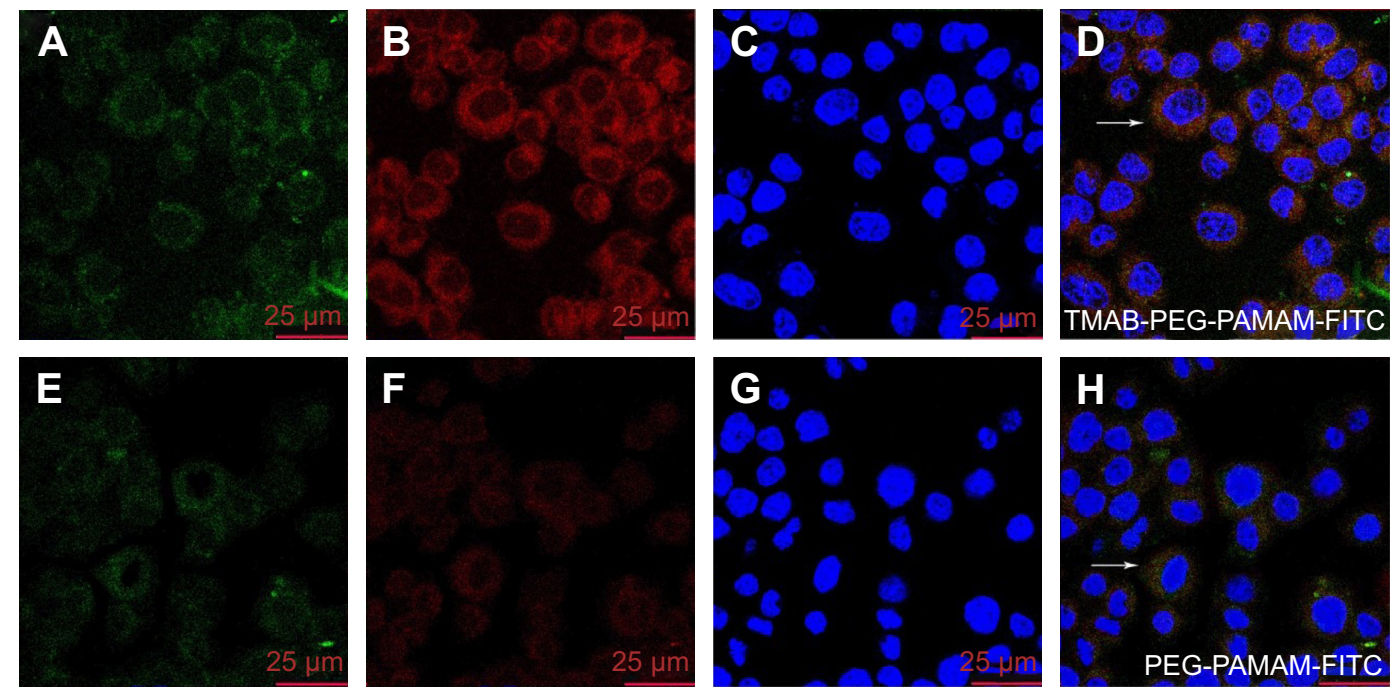

Figure 10 Confocal fluorescence microscopy images of TMAB-PEG-PAMAM-FITC (A-D) and PEG-PAMAM-FITC (E-H) bound to BT474 cells.

Notes: (A, E) FITC-labeled conjugates; (B, F) Lyso-Tracker red-labeled endo-lysosomes; (C, G) Hoechst-labeled nuclei; and (D, H) overlay of A, B, C and E, F, G. The white arrows indicate the conjugates colocalized with lysosome.

Abbreviations: TMAB, trastuzumab; PEG, polyethylene glycol; PAMAM, polyamidoamine; FITC, fluorescein isothiocyanate. 


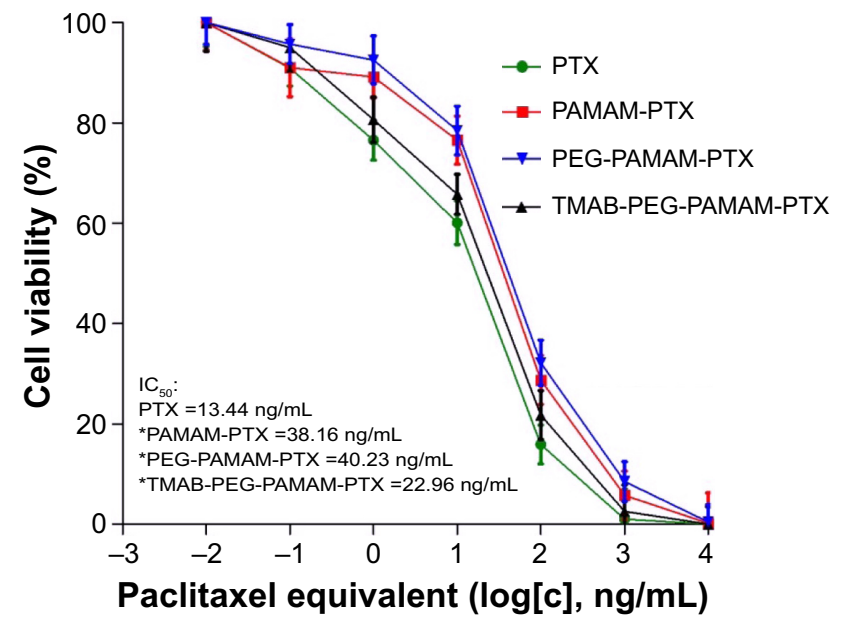

Figure I I In vitro cytotoxicity of free PTX, PAMAM-PTX, PEG-PAMAM-PTX, and TMAB-PEG-PAMAM-PTX conjugates toward HER2-positive BT474 breast cancer cells.

Notes: The data are presented as the means $\pm S D$. ${ }^{*} P<0.05$ compared to free paclitaxel.

Abbreviations: PTX, paclitaxel; PAMAM, polyamidoamine; PEG, polyethylene glycol; TMAB, trastuzumab; HER2, human epidermal growth factor 2; SD, standard deviation; $I_{50}$, half-maximal inhibitory concentration. liver tissue, followed by the tumor and spleen. The mean fluorescence intensity of the tumor tissue in the test group was the highest (approximately 1.5 times greater than the control group) among all of the tissues. This result further highlighted that the targeted conjugate preferentially accumulates in tumor tissues and targets breast cancer cells in a breast cancer mouse model. These results confirmed that the TMAB-PEG-PAMAM conjugate has significant promise in tumor-targeted drug delivery.

\section{Conclusion}

In summary, we successfully synthesized a HER2-targeted TMAB-PEG-PAMAM-PTX conjugate that exhibited a spherical shape and a narrow size distribution. This conjugate could specifically target cells and tumors that overexpress HER2. The in vitro cytotoxicity of the TMABfunctionalized PAMAM-PTX conjugate was lower than that of free PTX but possessed the advantage of alleviating side
A

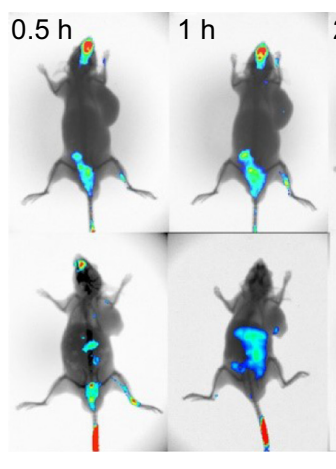

B
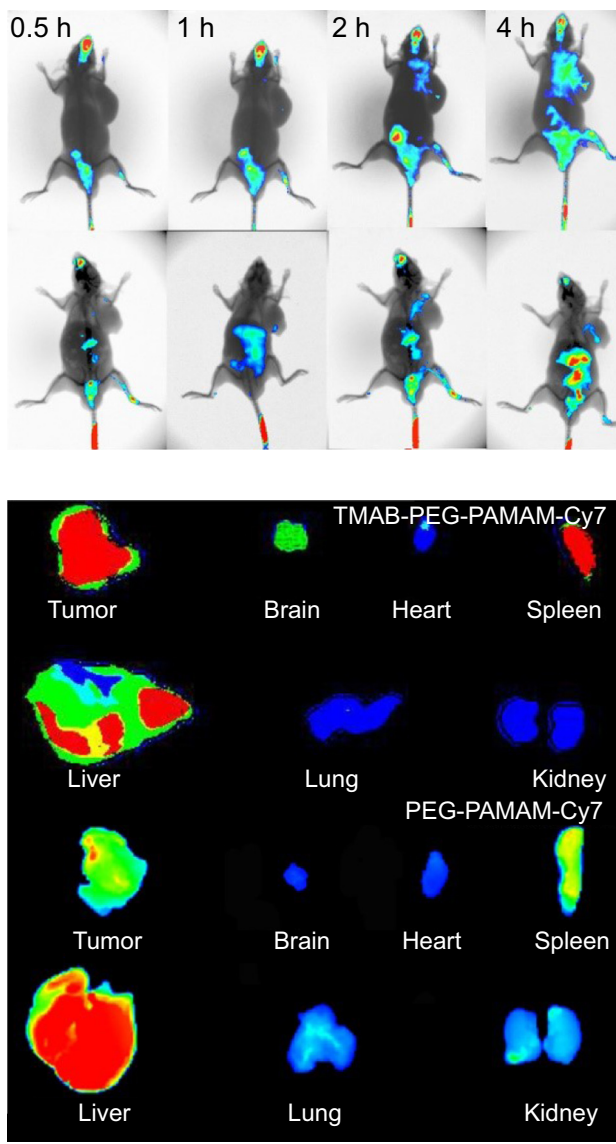
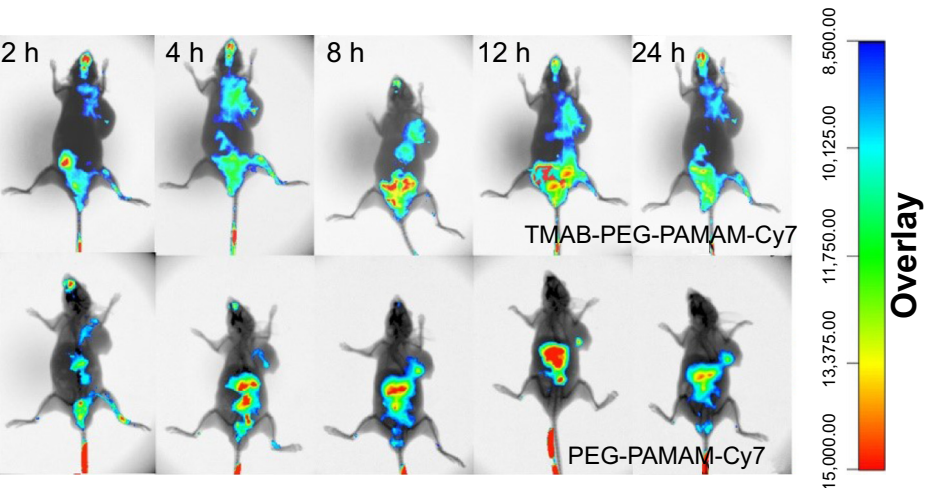

C

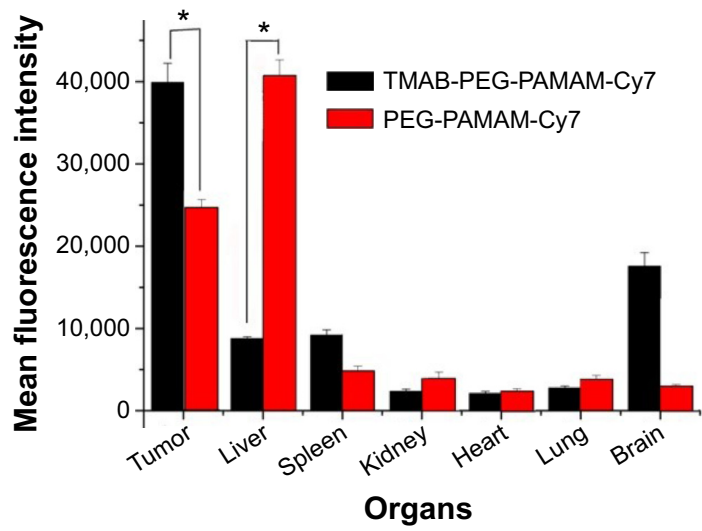

Figure 12 In vivo and ex vivo imaging of BT474 tumor-bearing mice.

Notes: (A) In vivo imaging of BT474 tumor-bearing mice at 0.5, I, 2, 4, 8, 12, and 24 hours after intravenous injection of PEG-PAMAM-Cy7 (control group) and TMABPEG-PAMAM-Cy7 (test group). (B) Ex vivo imaging of tumors and organs excised from BT474 tumor-bearing mice at 24 hours. (C) Analysis of the fluorescence signal (mean fluorescence intensity of the ROIs) in tumors and organs from ex vivo imaging at 24 hours. Experiments were carried out using $B$ T474 tumor-bearing nude mice ( $n=3$ ) when the tumor volume reached $250 \mathrm{~mm}^{3}$. The data are presented as the means $\pm S D(n=3)$. $* P<0.05$ compared to PEG-PAMAM-Cy7.

Abbreviations: PEG, polyethylene glycol; PAMAM, polyamidoamine; Cy7, cyanine7; TMAB, trastuzumab; SD, standard deviation; ROI, region of interest; h, hours. 
effects when applied in vivo because of its superior ability to target tumor tissues. In future studies, we will evaluate the therapeutic efficacy of this conjugate in animal models.

\section{Acknowledgments}

This work was supported by the Natural Science Foundation of Shandong (No: ZR2014HM062) and the Taishan Scholar Project ZiMei Wu.

\section{Disclosure}

The authors report no conflicts of interest in this work.

\section{References}

1. Shi P, Gustafson JA, MacKay JA. Genetically engineered nanocarriers for drug delivery. Int J Nanomedicine. 2014;9:1617-1626.

2. Sharma A, Jain N, Sareen R. Nanocarriers for diagnosis and targeting of breast cancer. Biomed Res Int. 2013;2013:960821.

3. Fay F, Scott CJ. Antibody targeted nanoparticles for cancer therapy. Immunotherapy. 2011;3(3):381-394.

4. Schroeder RL, Stevens CL, Sridhar J. Small molecule tyrosine kinase inhibitors of ErbB2/HER2/Neu in the treatment of aggressive breast cancer. Molecules. 2014;19(9):15196-15212.

5. Cirstoiu-Hapca A, Buchegger F, Lange N, Bossy L, Gurny R, Delie F. Benefit of anti-HER2-coated paclitaxel-loaded immuno-nanoparticles in the treatment of disseminated ovarian cancer: Therapeutic efficacy and biodistribution in mice. J Control Release. 2010;144(3):324-331.

6. Sun B, Ranganathan B, Feng SS. Multifunctional poly(D,L-lactide-coglycolide)/montmorillonite (PLGA/MMT) nanoparticles decorated by Trastuzumab for targeted chemotherapy of breast cancer. Biomaterials. 2008;29(4):475-486.

7. Yang T, Choi MK, Cui FD, et al. Antitumor effect of paclitaxel-loaded PEGylated immunoliposomes against human breast cancer cells. Pharm Res. 2007;24(12):2402-2411.

8. Patri AK, Kukowska-Latallo JF, Baker JR Jr. Targeted drug delivery with dendrimers: comparison of the release kinetics of covalently conjugated drug and non-covalent drug inclusion complex. Adv Drug Deliv Rev. 2005;57(15):2203-2214.

9. Cheng Y, Xu Z, Ma M, Xu T. Dendrimers as drug carriers: applications in different routes of drug administration. J Pharm Sci. 2008;97(1): 123-143.

10. Jain A, Dubey S, Kaushik A, Tyagi AK. Dendrimer: a complete drug carrier. Int J Pharm Sci Drug Res. 2010;1(4):38-52.

11. Svenson S, Chauhan AS. Dendrimers for enhanced drug solubilization. Nanomedicine (Lond). 2008;3(5):679-702.

12. Majoros IJ1, Myc A, Thomas T, Mehta CB, Baker JR Jr. PAMAM dendrimer-based multifuntional conjugate for cancer therapy: synthesis, characterization, and functionality. Biomacromolecules. 2005;7(2):572-579.

13. Wang W, Xiong W, Wan J, Sun X, Xu H, Yang X. The decrease of PAMAM dendrimer-induced cytotoxicity by PEGylation via attenuation of oxidative stress. Nanotechnology. 2009;20(10):105103.

14. Greg T Hermanson. Bioconjugate_Techniques-2nd_edtion. 2008

15. Lee JH, Nan A. Combination drug delivery approaches in metastatic breast cancer. J Drug Deliv. 2012;2012:915375.
16. Kaminskas LM, McLeod VM, Porter CJ, Boyd BJ. Association of chemotherapeutic drugs with dendrimer nanocarriers: an assessment of the merits of covalent conjugation compared to noncovalent encapsulation. Mol Pharm. 2012;9:355-373.

17. Cline EN, Li M-H, Choi SK, et al. Paclitaxel-conjugated PAMAM dendrimers adversely affect microtubule structure through two independent modes of action. Biomacromolecules. 2013;14(3):654-664.

18. Yellepeddi VK, Pisal DS, Kumar A, et al. Permeability of surfacemodified polyamidoamine (PAMAM) dendrimers across Caco-2 cell monolayers. Int J Pharm. 2008;350:113-121.

19. Luo D, Haverstick K, Beicheva N, Han E, Saltzman WM. Poly(ethylene glycol)-Conjugated PAMAM Dendrimer for Biocompatible, HighEfficiency DNA Delivery. Macromolecules. 2008;35:3456-3462.

20. Qi R, Gao Y, Tang Y, et al. PEG-conjugated PAMAM dendrimers mediate efficient intramuscular gene expression. AAPS J. 2009; 11(3):395-405.

21. Thomas PT, Shukla R, Kotlyar A, et al. Dendrimer-epidermal growth factor conjugate displays superagonist activity. Biomacromolecules. 2007;9(2):603-609.

22. Patri AK, Myc A, Beals J, Thomas TP, Bander NH, Baker JR Jr. Synthesis and in vitro testing of J591 antbody-denderimer conjugates for targeted prostate cancer therapy. Bioconjug Chem. 2004;15:1174-1181.

23. Pimpha N, Chaleawlert-umpon S, Chruewkamlow N, Kasinrerk W. Preparation of anti-CD4 monoclonal antibody-conjugated magnetic poly(glycidyl methacrylate) particles and their application on CD4+ lymphocyte separation. Talanta. 2011;84(1):89-97.

24. Davis ME, Chen Z, Shin DM. Nanoparticle therapeutics: an emerging treatment modality for cancer. Nat Rev Drug Discov. 2008;7(9): 771-782.

25. Sawant RR, Torchilin VP. Challenges in development of targeted liposomal therapeutics. AAPS J. 2012;14(2):303-315.

26. Jin J, Sui B, Gou J, et al. PSMA ligand conjugated PCL-PEG polymeric micelles targeted to prostate cancer cells. PLoS One. 2014;9(11): $1-12$.

27. Vicente-Alique E, Núñez-Ramírez R, Vega JF, Hu P, Martínez-Salazar J. Size and conformational features of ErbB2 and ErbB3 receptors: a TEM and DLS comparative study. Eur Biophys J. 2011;40(7):835-842.

28. Julien DC, Behnke S, Wang G, Murdoch GK, Hill RA. Utilization of monoclonal antibody-targeted nanomaterials in the treatment of cancer. MAbs. 2011;3(5):467-478.

29. Zhu S, Hong M, Zhang L, Tang G, Jiang Y, Pei Y. PEGylated PAMAM dendrimer-doxorubicin conjugates: in vitro evaluation and in vivo tumor accumulation. Pharm Res. 2010;27(1):161-174.

30. Zheng N, Dai W, Du W, et al. A novel lanreotide-encoded micelle system targets paclitaxel to the tumors with overexpression of somatostatin receptors. Mol Pharm. 2012;9(5):1175-1188.

31. Hussain AF, Krüger HR, Kampmeier F, et al. Targeted delivery of dendritic polyglycerol-doxorubicin conjugates by $\mathrm{scFv}$-SNAP fusion protein suppresses EGFR+ cancer cell growth. Biomacromolecules. 2013; 14(8):2510-2520.

32. Yu Y, Wang ZH, Zhang L, et al. Mitochondrial targeting topotecan-loaded liposomes for treating drug-resistant breast cancer and inhibiting invasive metastases of melanoma. Biomaterials. 2012;33(6):1808-1820.

33. Menjoge AR, Kannan RM, Tomalia DA. Dendrimer-based drug and imaging conjugates: design considerations for nanomedical applications. Drug Discov Today. 2010;15(5-6):171-185. 


\section{Supplementary materials}

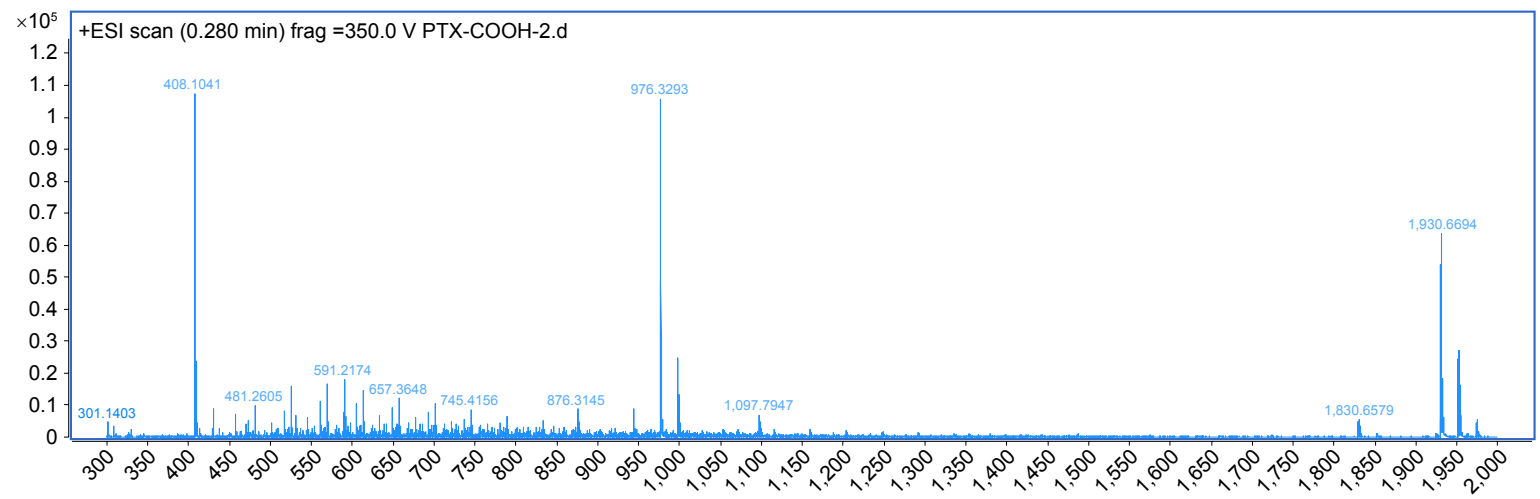

Counts vs mass-to-charge $(\mathrm{m} / \mathrm{z})$

Figure SI Electrospray ionization mass spectra (ESI-MS) of PTX-2'-hemisuccinate.

Abbreviations: min, minutes; frag, fragments; PTX, paclitaxel; vs, versus.

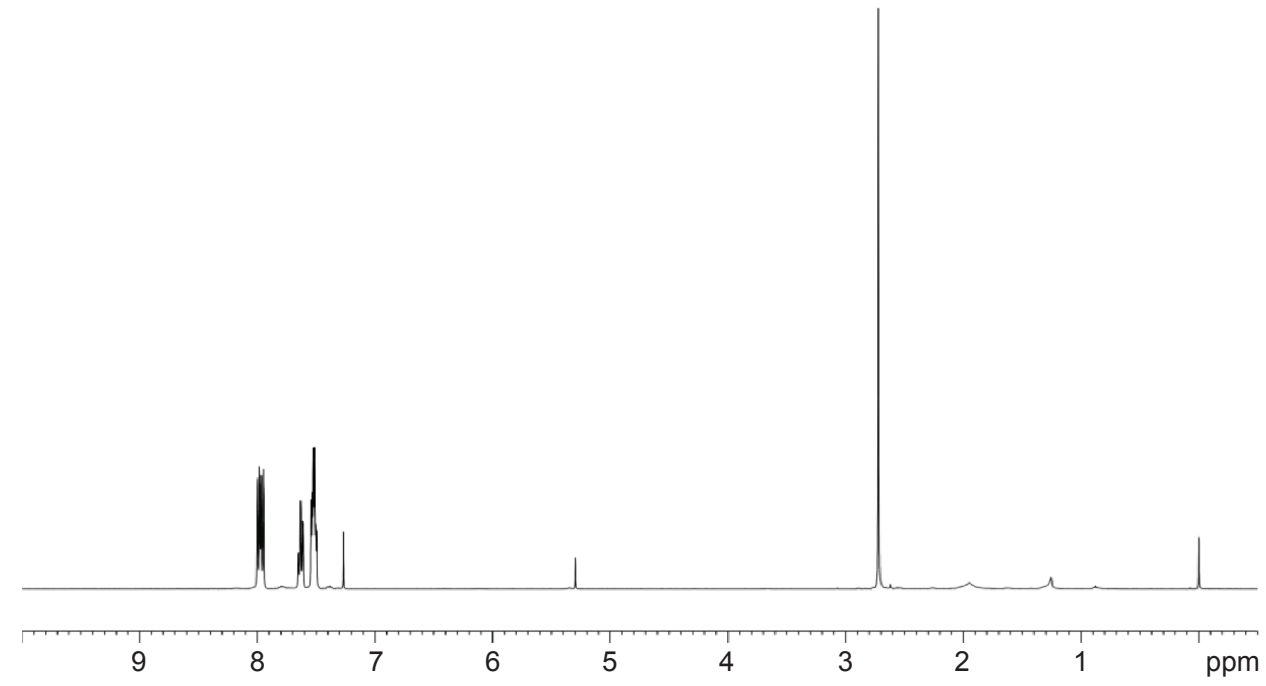

Figure S2 ' $\mathrm{H}-\mathrm{NMR}$ of SDPP in $\mathrm{CCl}_{3} \mathrm{D}$ at $400 \mathrm{MHz}$.

Abbreviations: 'H-NMR, proton nuclear magnetic resonance; SDPP, N-hydroxysuccinimide diethyl phosphate.

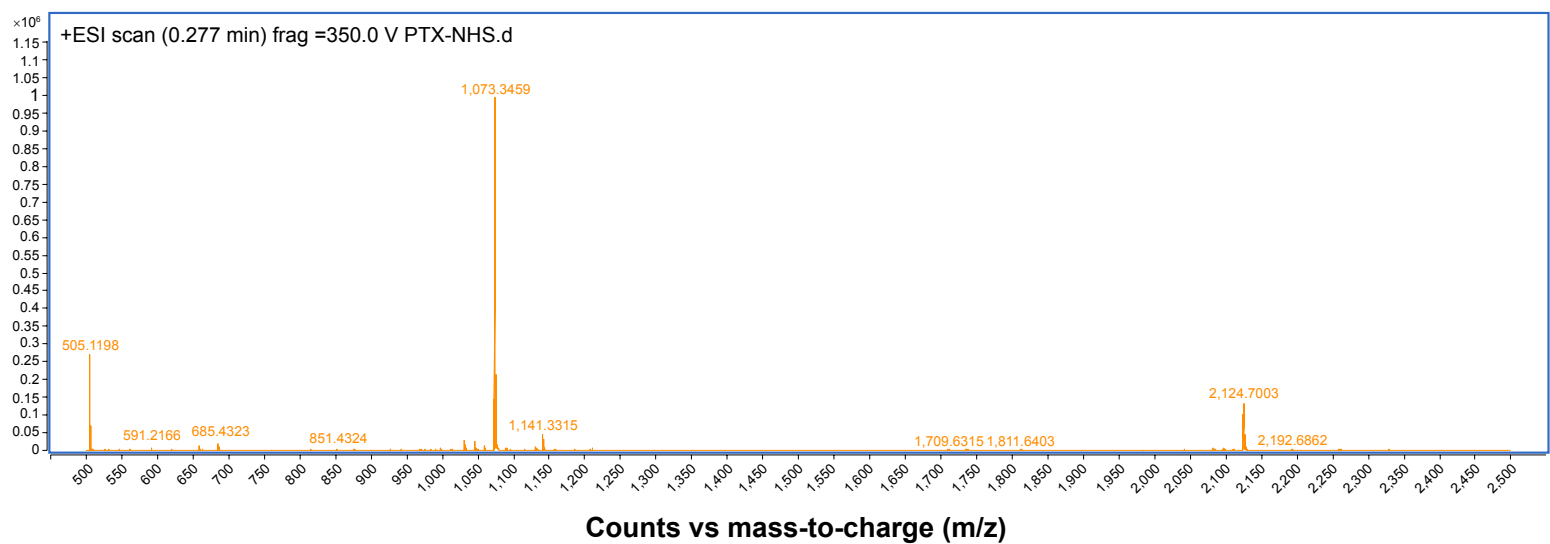

Figure S3 Electrospray ionization mass spectra (ESI-MS) of PTX-NHS.

Abbreviations: PTX, paclitaxel; NHS, N-hydroxysuccinimide; min, minutes; frag, fragments; vs, versus. 

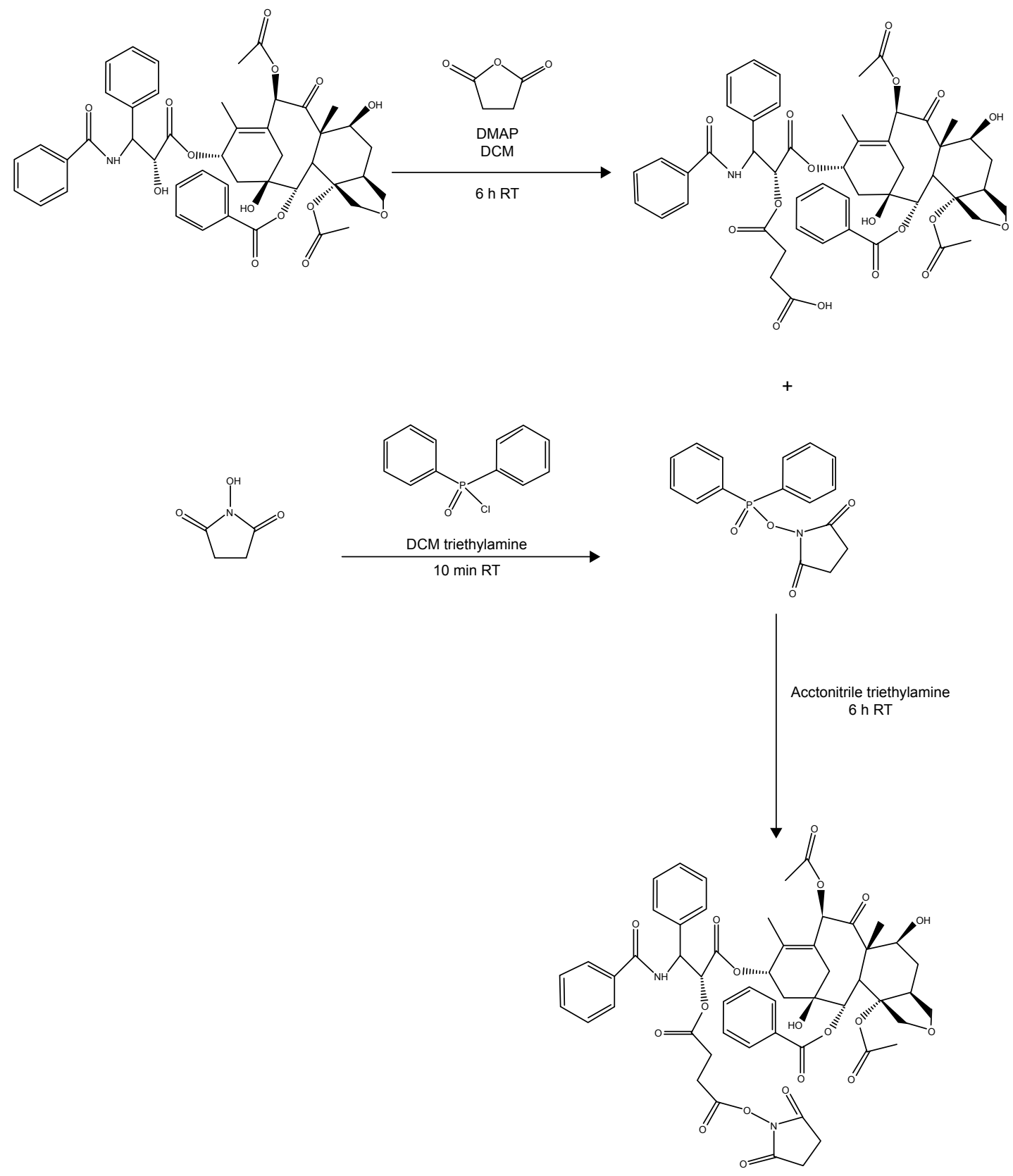

Figure S4 Schematic synthesis of PTX-NHS.

Abbreviations: h, hours; min, minutes; PTX, paclitaxel; NHS, N-hydroxysuccinimide; DMAP, 4-dimethylaminopyridine; RT, room temperature; DCM, dichloromethane. 


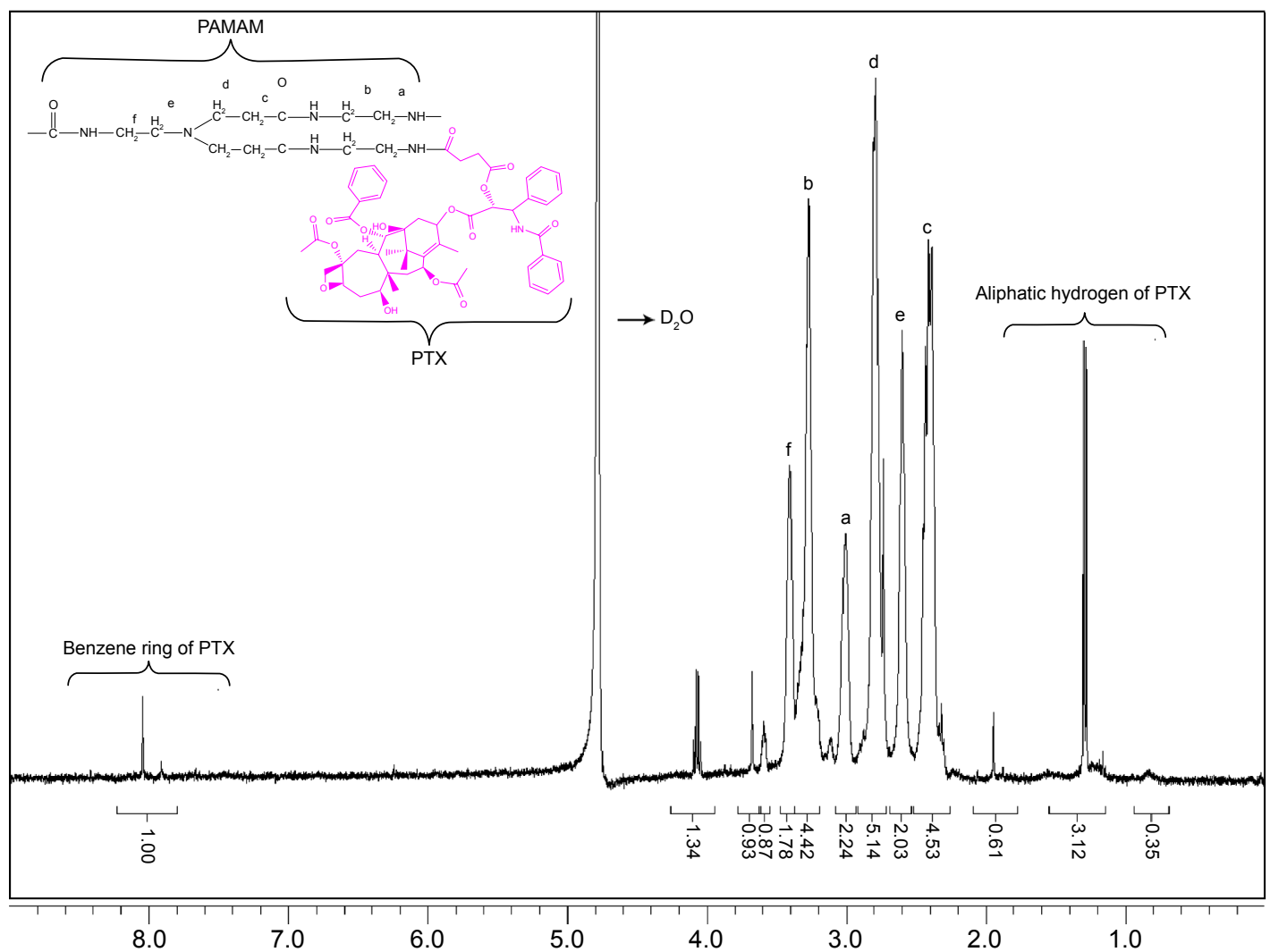

Figure S5 Proton nuclear magnetic resonance ('H-NMR) spectra of PAMAM-PTX in $\mathrm{D}_{2} \mathrm{O}$ at $400 \mathrm{MHz}$.

Note: $a-f$ represent the protons of PAMAM.

Abbreviations: PAMAM, polyamidoamine; PTX, paclitaxel.

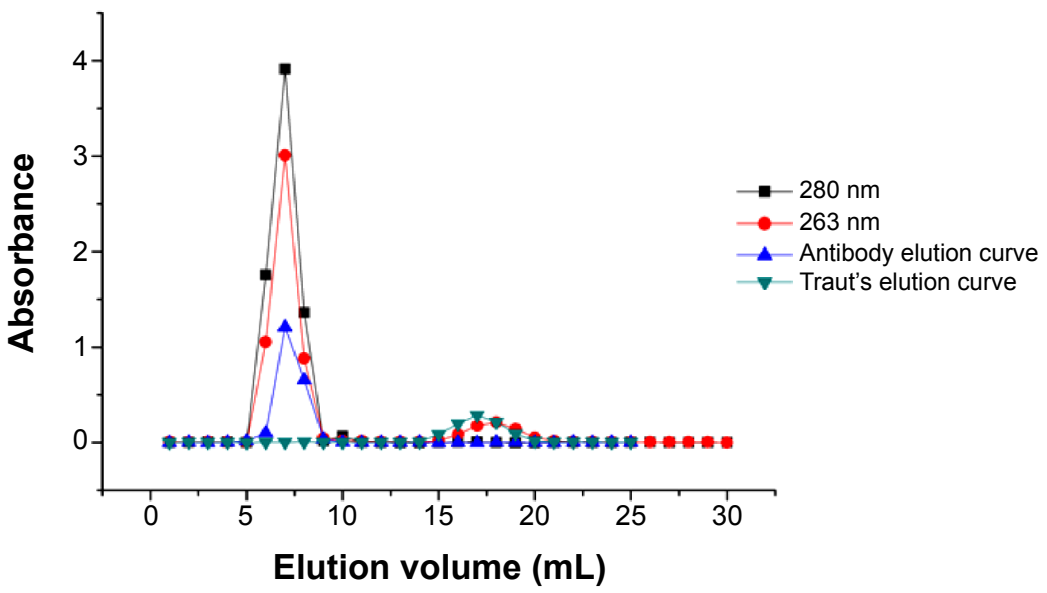

Figure S6 The elution curve of thiolated TMAB and Traut's reagent.

Abbreviation: TMAB, trastuzumab.

International Journal of Nanomedicine

\section{Publish your work in this journal}

The International Journal of Nanomedicine is an international, peerreviewed journal focusing on the application of nanotechnology in diagnostics, therapeutics, and drug delivery systems throughout the biomedical field. This journal is indexed on PubMed Central, MedLine, CAS, SciSearch $\AA$, Current Contents $\AA /$ Clinical Medicine,
Journal Citation Reports/Science Edition, EMBase, Scopus and the Elsevier Bibliographic databases. The manuscript management system is completely online and includes a very quick and fair peer-review system, which is all easy to use. Visit http://www.dovepress.com/ testimonials.php to read real quotes from published authors. 